\title{
Dietary Factors and Modulation of Bacteria Strains of Akkermansia muciniphila and Faecalibacterium prausnitzii: A Systematic Review
}

\author{
Sanne Verhoog ${ }^{1, *(1)}$, Petek Eylul Taneri ${ }^{2}$, Zayne M. Roa Díaz ${ }^{1}\left(\mathbb{D}\right.$, Pedro Marques-Vidal $^{3}{ }^{(D)}$, \\ John P. Troup ${ }^{4}$, Lia Bally ${ }^{5}$, Oscar H. Franco ${ }^{1}$, Marija Glisic ${ }^{6}$ (i) and Taulant Muka ${ }^{1}$ \\ 1 Institute of Social and Preventive Medicine, University of Bern, 3012 Bern, Switzerland \\ 2 Corlu Cancer Early Diognosis and Training Center, 59100 Tekirdag, Turkey \\ 3 Department of Medicine, Internal Medicine, Lausanne University Hospital (CHUV), \\ 1011 Lausanne, Switzerland \\ 4 Standard Process Inc Nutrition Innovation Center, Kannapolis, NC 28018, USA \\ 5 Department of Diabetes, Endocrinology, Clinical Nutrition and Metabolism, Bern University Hospital, \\ 3010 Bern, Switzerland \\ 6 Leibniz Institute for Prevention Research and Epidemiology-BIPS, 28359 Bremen, Germany \\ * Correspondence: sanne.verhoog@ispm.unibe.ch; Tel.: +41-31-631-56-75
}

Received: 6 June 2019; Accepted: 8 July 2019; Published: 11 July 2019

\begin{abstract}
Akkermansia muciniphila and Faecalibacterium prausnitzii are highly abundant human gut microbes in healthy individuals, and reduced levels are associated with inflammation and alterations of metabolic processes involved in the development of type 2 diabetes. Dietary factors can influence the abundance of A. muciniphila and F. prausnitzii, but the evidence is not clear. We systematically searched PubMed and Embase to identify clinical trials investigating any dietary intervention in relation to A. muciniphila and F. prausnitzii. Overall, 29 unique trials were included, of which five examined A. muciniphila, 19 examined F. prausnitzii, and six examined both, in a total of 1444 participants. A caloric restriction diet and supplementation with pomegranate extract, resveratrol, polydextrose, yeast fermentate, sodium butyrate, and inulin increased the abundance of $A$. muciniphila, while a diet low in fermentable oligosaccharides, disaccharides, monosaccharides, and polyols decreased the abundance of $A$. muciniphila. For F. prausnitzii, the main studied intervention was prebiotics (e.g. fructo-oligosaccharides, inulin type fructans, raffinose); seven studies reported an increase after prebiotic intervention, while two studies reported a decrease, and four studies reported no difference. Current evidence suggests that some dietary factors may influence the abundance of A. muciniphila and F. prausnitzii. However, more research is needed to support these microflora strains as targets of microbiome shifts with dietary intervention and their use as medical nutrition therapy in prevention and management of chronic disease.
\end{abstract}

Keywords: Akkermansia muciniphila; Faecalibacterium prausnitzii; microbiome; systematic review; randomized controlled trials; dietary interventions

\section{Introduction}

The gut microbiota, defined as the complex, diverse, and vast microbial community resided in the human gut, is emerging as a key player in the pathophysiology of several chronic conditions [1]. Gut microbiota extract energy from nutrients and regulate several biological processes. A microbiota imbalance, such as low abundance of Akkermansia muciniphila and Faecalibacterium prausnitzii, favours inflammatory processes, potentially leading to inflammatory disorders of the gastrointestinal tract such as irritable bowel syndrome and inflammatory bowel disease, as well as to colorectal cancer [2-6]. 
Also, increases in A. muciniphila and F. prausnitzii abundance can regulate metabolic functions and appear to exert protective effects against the development of obesity [7], type 2 diabetes (T2D) [8], and atherosclerosis [9]. Therefore, these two bacteria have been considered as potential bioindicators of human cardiometabolic health and conditions where underlying inflammation plays a role. A. muciniphila is a mucin-degrading bacterium of the phylum Verrucomicrobia, while F. prausnitzii is an important butyrate-producer of the phylum of Firmicutes. F. prausnitzii is also the major bacterium of the Clostridium leptum group [10,11]. It accounts for $5 \%$ of total bacteria in faeces and it is an important source of energy for the colonocytes [12,13].

Modulating the abundance of A. muciniphila and F. prausnitzii in the intestinal flora may contribute to prevent and treat inflammatory and cardiometabolic diseases [14-16]. Studies have shown that dietary factors can influence the diversity and composition of the gut microbiome more than human genetic factors [17]. Data from animal studies suggest that polyphenols-rich diets and specific phytochemicals such as curcumin and epigallocatechin gallate may improve the abundance of A. muciniphila and F. prausnitzii in the gut microbiome [18-22]. A clinical trial with obese insulin-resistant patients showed that resveratrol, a natural phenol, increased the abundance of $A$. muciniphila in Caucasians but not in other ethnic groups [23]. A diet high in fermentable oligo-, di- and monosaccharides and polyphenols (FODMAP), which has been implicated in digestive diseases such as irritable bowel syndrome, inflammatory bowel disease, and Crohn's disease, resulted in changes in gut microbiota including changes in A. muciniphila and F. prausnitzii but the evidence is not consistent [24,25]. Furthermore, intake of dietary fibres, such as the soluble fibre inulin, has been associated with increased abundance of A. muciniphila and F. prausnitzii in healthy and overweight/obese individuals, but another study showed no influence of inulin intake on F. prausnitzii [26-28]. Despite the emerging evidence of the impact of dietary factors on changing the microbiome composition, a comprehensive assessment of the dietary interventions in modulating A. muciniphila and F. prausnitzii is lacking. Identifying dietary microbiome modulators may help understanding how diet and phytochemicals can be employed in medical nutrition therapy to increase the abundance of A. muciniphila and F. prausnitzii.

Therefore, the aim of this systematic review is to identify, integrate and discuss all available evidence from clinical trials in humans on the association of diet with changes in A. muciniphila and F. prausnitzii.

\section{Material and Methods}

\subsection{Literature Search}

This review was conducted and reported in accordance with the PRISMA [29] and MOOSE [30] guidelines (Tables S1 and S2). PubMed and Embase were used to identify published studies from database inception until April 23, 2019 (date last searched) that examined the associations between any dietary factor and changes in A. muciniphila and F. prausnitzii in humans. To capture all relevant studies, we used a broad search strategy using terms related to A. muciniphila and F. prausnitzii (Appendix A). We did not apply any restrictions with regard to language and date. Furthermore, to identify additional relevant studies, we checked the reference lists of the studies included in the current review.

\subsection{Study Selection Criteria}

To be included in the review, studies had to full-fill all of the following criteria: (i) were clinical trials (randomized placebo-controlled trials, randomized trials comparing different exposures and single-arm trials); (ii) included subjects $18+$ years, and (iii) reported associations between any dietary intervention and absolute and relative abundance of A. muciniphila, or F. prausnitzii, or both. As we were interested to understand the effect of dietary factors on gut microbiome, we decided to include all trials irrespective of their aim; therefore, all eligible trials that reported baseline and end of study information on outcomes of interest were included in this review. Studies conducted in animals, children/teenagers, conference abstracts, letters to the editor, interventions examining probiotics 
solely and non-interventional studies were excluded. Following the selection criteria, two reviewers independently screened titles and abstracts for eligibility. Next, two reviewers assessed the full-text of potentially eligible studies. In cases of disagreement, a decision was made by consensus or, if necessary, a third reviewer was consulted.

\subsection{Data Extraction}

Two reviewers extracted the data independently using a predesigned form including study design, study population, location, age range, dietary intervention and control (if applicable), duration of intervention and study results.

\subsection{Assessing the Quality of Trials}

Two reviewers evaluated the risk of bias within each study using "The Cochrane Collaboration's tool" [31]. Studies were judged to be at low or high risk of bias based on criteria to evaluate random sequence generation, allocation concealment, blinding of participants/personnel and outcome assessment, incomplete outcome data and selective reporting. Trials were considered to be in low risk of bias if allocation concealment, blinding of participants and outcome assessors were all coded "yes", if a compliance assessment was done and the number of dropouts and reasons for dropout were reported; otherwise, the trials were considered to be at high risk of bias. If the risk of bias could not be determined in any of the segments, (e.g., information not provided) the risk of bias was classified as unknown. Lastly, for each trial, an overall score of quality was provided. Good quality was assigned when all criteria were met (i.e. low risk of bias for each domain). Fair quality was assigned when one criterion was not met (i.e. high risk of bias for one domain) or two criteria were unclear, and the assessment that this was unlikely to have biased the outcome, and there is no known important limitation that could invalidate the results. Poor quality was assigned when (i) one criterion was not met or two criteria were unclear, and the assessment that this was likely to have biased the outcome, and there are important limitations that could invalidate the results, or (ii) when two or more criteria were listed as high or unclear risk of bias.

\section{Results}

\subsection{Study Identification and Selection}

In total, we identified 1433 relevant citations. After screening based on titles and abstracts, 59 citations were selected for detailed full text evaluation. Of those, 30 articles [23-28,32-55], based on 29 unique trials, met the selection criteria and were included in the review (Figure 1). Among these, 19 articles examined $F$. prausnitzii $[26,27,32,34,36-39,41,42,44,46,47,49-52,54,55]$, five articles examined A. muciniphila $[23,28,35,43,48]$, and six articles examined both $[24,25,33,40,45,53]$. 
384 potentially relevant citations identified in Pubmed

1049 potentially relevant citations identified in Embase

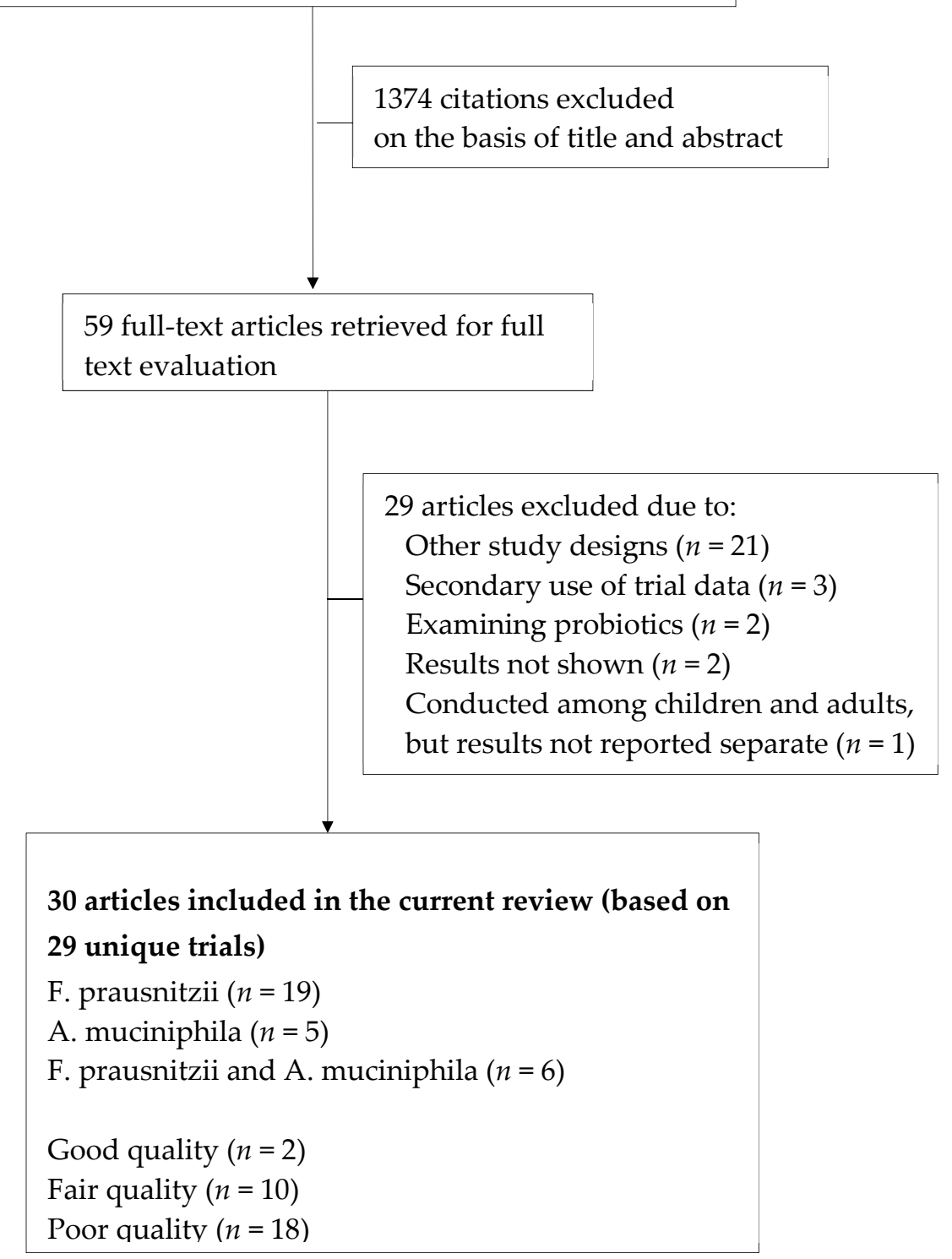

Figure 1. Flowchart of studies included in the current review.

\subsection{Characteristics of the Included Trials}

Of the 29 unique included trials, 15 were conducted in Europe, 8 in North America, 4 in Australia and New Zealand, 1 in Iran, and 1 in China; 22 were conducted among both men and women, 4 among men and 3 among women. Overall, the trials reported results for 1444 participants. All trials were published after 2005 and 80 percent in the last 5 years. The baseline age of the participants was $18+$. The duration of the interventions ranged from 2 weeks to 6 months. The design of the included trials comprised randomized double-blind placebo-controlled trials, single-arm trials, multi-arm parallel trials, cross-over trials, and single and multi-centre trials. Detailed characteristics of the included trials are presented in Tables 1 and 2. Two trials scored an overall good quality score, indicating a low risk of bias in all domains (Table S3), whereas for the other trials the overall score was fair $(n=10)$ or poor $(n=18)$. There was a low risk of bias for all trials in the domain sequence generation and allocation concealment. For five trials, the risk of bias in the domain blinding of participants and personnel was unclear. In the domain blinding of assessment, 16 trials had an unclear risk of bias, as this was not clearly stated in these trials. Nine trials did not report how they handled missing data, and were 
therefore classified as being unclear in the assessment of risk of bias in the domain of missing data. High risk of bias was found in the domain of selective reporting for 14 trials, as they did not report effect estimates. The domain "other bias" included the risk of false positive findings for 14 trials, as these trials did not account for multiple testing in their analysis.

\subsection{Trials Examining A. muciniphila}

Eleven trials examined the association between a dietary intervention and A. muciniphila [23-25, $28,33,35,40,43,45,48,53]$. The dietary interventions examined were a caloric restriction diet, a reduced energy diet, a diet low in fermentable oligo-, di-, mono-saccharides and polyols (FODMAP), supplemental fibres, a yeast fermentate (EpiCor), sodium butyrare and inulin, pomegranate extract, kiwifruit capsules, and resveratrol. Findings are summarized in Table 1.

Dao $\mathrm{M}$ et al. evaluated a caloric restriction diet compared to a weight stabilization diet among overweight and obese participants [35]. In the caloric restricted group, the abundance of A. muciniphila decreased in participants with a high baseline level of $A$. muciniphila and increased in participants with low A. muciniphila levels. In the weight stabilization diet group, the abundance of A. muciniphila decreased in participants with both low and high baseline levels of A. muciniphila. Similarly to Dao $M$ et al. [13], Medina-Vera I et al. examined a reduced energy diet compared to placebo in patients with T2D [45]. They reported that consumption of the reduced-energy diet increased levels of A. muciniphila by approximately $125 \%$. However, two other studies coming from the same cross-over trial examined a diet low in FODMAPs compared to a typical Australian diet containing FODMAPs, and showed that the typical Australian diet increased absolute and relative abundance of A. muciniphila $[24,25]$.

A cross-over trial conducted showed that polydextrose supplementation increased A. muciniphila compared to soluble corn fibre supplementation or placebo (i.e. no supplemental fibre control) among healthy individuals [40]. Another cross-over trial reported no changes in relative or absolute abundance of $A$. muciniphila after increasing the intake of resistant starch and wheat bran foods among healthy individuals and patients with ulcerative colitis in remission [53]. Supplementation with EpiCor, a yeast fermentate, increased A. muciniphila compared to placebo (maltodextrin) in healthy individuals with moderate symptoms of gastrointestinal discomfort [48]. Supplementation with sodium butyrate or inulin increased A. muciniphila compared to placebo (starch) in overweight and obese diabetic patients, but no significant differences were found with combined sodium butyrate and inulin supplementation [28].

Pomegranate extract increased A. muciniphila by 47 -fold in healthy metabolite urolithin A producers compared to non-producers [43]. However, kiwifruit capsules did not have a significant effect on A. muciniphila abundance in healthy and functionally constipated individuals [33]. Resveratrol supplementation led to an increase in A. muciniphila in USA Caucasians, but not in other ethnic groups [23].

\subsection{Trials Examining F. prausnitzii}

Twenty-five studies examined the effect of dietary interventions on F. prausnitzii [24-27,3234,36-42,44-47,49-55]. Of these 25 studies, 17 studies examined prebiotics and the other studies examined isoflavones, dietary fat, carbohydrates, a ketogenic diet, kiwifruit capsules, sun-dried raisins, iron therapy, and a Chinese herbal formula. The findings are summarized in Table 2. 
Table 1. Descriptive Summary of Randomized Clinical Trials Investigating the Associations Between Dietary Interventions and Akkermansia Muciniphila.

\begin{tabular}{|c|c|c|c|c|c|c|c|c|c|}
\hline $\begin{array}{l}\text { Lead Author, } \\
\text { Publication Year }\end{array}$ & Study Design & $\begin{array}{l}\text { Location/Age } \\
\text { Range }\end{array}$ & $\begin{array}{l}\text { Individual Health } \\
\text { Status }\end{array}$ & $\begin{array}{l}\text { Total } \\
\text { Participants }\end{array}$ & Sex & $\begin{array}{l}\text { Period of } \\
\text { Intervention }\end{array}$ & $\begin{array}{l}\text { Dietary Treatment Chara } \\
\text { Intervention Type }\end{array}$ & $\begin{array}{l}\text { cteristics } \\
\text { Control Type }\end{array}$ & Main Findings \\
\hline \multirow[t]{2}{*}{$\begin{array}{l}\text { Blatchford P } \\
\text { et al. 2017* [1] }\end{array}$} & $\begin{array}{l}\text { Randomized } \\
\text { double-blind } \\
\text { placebo-controlled } \\
\text { cross-over trial }\end{array}$ & $\begin{array}{l}\text { New Zealand/ } \\
23-56\end{array}$ & $\begin{array}{l}\text { Healthy participants } \\
\text { with no clinical } \\
\text { symptoms of } \\
\text { constipation and } \\
\text { functionally } \\
\text { constipated } \\
\text { participants }\end{array}$ & 29 & $\mathrm{~W}$ and $\mathrm{M}$ & $\begin{array}{l}4 \text { weeks each } \\
\text { intervention }(2 \text { weeks } \\
\text { washout period } \\
\text { between each } \\
\text { intervention) }\end{array}$ & $\begin{array}{l}\text { ACTAZINTM }(600 \mathrm{mg} / \mathrm{d}) \\
\text { green kiwifruit extract } \\
\text { low dose } \\
\text { ACTAZIN } \\
(2400 \mathrm{mg} / \mathrm{d}) \text { green } \\
\text { kiwifruit extract high } \\
\text { dose } \\
\text { Livaux } \\
\text { gold kiwifruit extract }\end{array}$ & $\begin{array}{l}\text { Placebo (isomalt } \\
\text { coloured green } \\
2400 \mathrm{mg} / \mathrm{d} \text { ) }\end{array}$ & $\begin{array}{l}\text { A. muciniphila was significantly more } \\
\text { abundant in the functionally } \\
\text { constipated group, but no effect of the } \\
\text { interventions on A. muciniphila. }\end{array}$ \\
\hline & $\begin{array}{l}\text { Single-arm } \\
\text { cross-over trial }\end{array}$ & $\begin{array}{l}\text { France/ } \\
41.9 \pm 12.3\end{array}$ & $\begin{array}{l}\text { Overweight and } \\
\text { obese participants }\end{array}$ & 49 & $\mathrm{~W}$ and $\mathrm{M}$ & 12 weeks & $\begin{array}{l}\text { Caloric restriction diet } \\
(1200 \mathrm{kcal} / \mathrm{d} \text { for } W \text { and } \\
1500 \mathrm{kcal} / \mathrm{d} \text { for M })\end{array}$ & $\begin{array}{l}\text { Weight stabilization } \\
\text { diet (prescribed } \\
\text { individually by a }\end{array}$ & $\begin{array}{l}\text { Caloric restriction diet: Subjects with } \\
\text { A. muciniphila at or above the median } \\
\text { had a decrease in abundance of } A \text {. } \\
\text { muciniphila while in the group with } A \text {. } \\
\text { muciniphila lower than median there } \\
\text { was an increase. The difference was } \\
\text { statistical significant. }\end{array}$ \\
\hline & & & & & & & & & $\begin{array}{l}\text { Weight stabilization diet: In both } \\
\text { subjects with } A \text {. muciniphila at or } \\
\text { above and lower, there was a decrease } \\
\text { in abundance of } A \text {. muciniphila with no } \\
\text { difference between the groups. }\end{array}$ \\
\hline $\begin{array}{l}\text { Halmos EP } \\
\text { et al. 2015* [16] }\end{array}$ & $\begin{array}{l}\text { Single-arm blinded } \\
\text { randomized } \\
\text { cross-over trial }\end{array}$ & Australia/18+ & $\begin{array}{l}\text { Healthy participants } \\
\text { and participants with } \\
\text { irritable bowel } \\
\text { syndrome }\end{array}$ & 33 & $\mathrm{~W}$ and $\mathrm{M}$ & 6 weeks & Diet low in FODMAPs & $\begin{array}{l}\text { Diet containing } \\
\text { FODMAP content of a } \\
\text { typical Australian diet }\end{array}$ & $\begin{array}{l}\text { Typical Australian diet increased } \\
\text { absolute and relative abundance for } \\
\text { mucus-associated A. muciniphila } \\
(p<0.001) \text {. }\end{array}$ \\
\hline $\begin{array}{l}\text { Halmos EP et al. } \\
2016^{*}[17]\end{array}$ & $\begin{array}{l}\text { Single-arm blinded } \\
\text { randomized } \\
\text { cross-over trial }\end{array}$ & Australia/18+ & $\begin{array}{l}\text { Patients with } \\
\text { clinically quiescent } \\
\text { Crohn's disease }\end{array}$ & 9 & $\mathrm{~W}$ and $\mathrm{M}$ & 6 weeks & Diet low in FODMAPs & $\begin{array}{l}\text { Diet containing } \\
\text { FODMAP content of a } \\
\text { typical Australian diet }\end{array}$ & $\begin{array}{l}\text { Relative abundance was higher for } \\
\text { mucus-associated } A \text {. muciniphila } \\
\text { during the Australian compared with } \\
\text { low FODMAP diet }(p=0.016) \text {. }\end{array}$ \\
\hline $\begin{array}{l}\text { Hooda S et al. } \\
2012^{*}[6]\end{array}$ & $\begin{array}{l}\text { Randomized } \\
\text { double-blind } \\
\text { placebo-controlled } \\
\text { cross-over trial }\end{array}$ & $\begin{array}{l}\text { USA/ } \\
27.5 \pm 4.33\end{array}$ & Healthy participants & 25 & $\mathrm{M}$ & 9 weeks & $\begin{array}{l}\text { Polydextrose (PDX) } \\
\text { (7 g, } 3 \text { times per day) } \\
\text { Soluble corn fiber (SCF) } \\
\text { (7 g, } 3 \text { times per day) } \\
\text { 'Low resistant starch } \\
\text { (RS)/wheat bran (WB)' }\end{array}$ & $\begin{array}{l}\text { Placebo (no } \\
\text { supplemental fiber } \\
\text { control (NFC) }(0 \mathrm{~g}, \\
3 \text { times per day)) }\end{array}$ & $\begin{array}{l}\text { A. muciniphila was greater after PDX } \\
\text { intake than after the NFC or SCF } \\
\text { treatment }(p<0.05) \text {. }\end{array}$ \\
\hline $\begin{array}{l}\text { James SL et al. } \\
2015^{*}[54]\end{array}$ & $\begin{array}{l}\text { Randomized } \\
\text { single-blind } \\
\text { cross-over trial }\end{array}$ & $\begin{array}{l}\text { Australia/ } \\
18-72\end{array}$ & $\begin{array}{l}\text { Patients with UC in } \\
\text { remission and healthy } \\
\text { subjects }\end{array}$ & 29 & $\mathrm{~W}$ and $\mathrm{M}$ & 8 weeks & $\begin{array}{l}\text { foods containing } 2-5 \mathrm{~g} \\
\mathrm{RS} \text { and } \\
2-5 \mathrm{~g} \text { WB fibre per day } \\
\text { 'High RS/WB' foods } \\
\text { containing } 15 \mathrm{~g} \\
\text { RS and } 12 \mathrm{~g} \text { WB fibre } \\
\text { per day }\end{array}$ & NA & $\begin{array}{l}\text { Patients with UC had a lower } \\
\text { abundance of } A \text {. muciniphila. For both } \\
\text { cohorts, increasing the intake of } \\
\text { RS/WB gave no indication of changes } \\
\text { in relative or absolute abundance. }\end{array}$ \\
\hline
\end{tabular}


Table 1. Cont.

\begin{tabular}{|c|c|c|c|c|c|c|c|c|c|}
\hline $\begin{array}{l}\text { Lead Author, } \\
\text { Publication Year }\end{array}$ & Study Design & $\begin{array}{l}\text { Location/Age } \\
\text { Range }\end{array}$ & $\begin{array}{l}\text { Individual Health } \\
\text { Status }\end{array}$ & $\begin{array}{l}\text { Total } \\
\text { Participants }\end{array}$ & Sex & $\begin{array}{l}\text { Period of } \\
\text { Intervention }\end{array}$ & $\begin{array}{l}\text { Dietary Treatment Char } \\
\text { Intervention Type }\end{array}$ & $\begin{array}{l}\text { cteristics } \\
\text { Control Type }\end{array}$ & Main Findings \\
\hline $\begin{array}{l}\text { Li Z } \\
\text { et al. } 2015 \text { [20] }\end{array}$ & Single-arm trial & USA/28.9 \pm 8 & Healthy volunteers & 20 & $\mathrm{~W}$ and $\mathrm{M}$ & 4 weeks & $\begin{array}{l}\text { Pomegranate extract } \\
(1000 \mathrm{mg})\end{array}$ & NA & $\begin{array}{l}\text { The data were not shown for the } \\
\text { overall population. A muciniphila was } \\
33 \text { (at baseline) and } 47 \text { fold (after } \\
4 \text { weeks) higher in stool samples of } \\
\text { Urolithin A producers compared to } \\
\text { non-producers. }\end{array}$ \\
\hline $\begin{array}{l}\text { Medina-Vera I } \\
\text { et al. 2019* [9] }\end{array}$ & $\begin{array}{l}\text { Randomized, } \\
\text { double-blind } \\
\text { placebo-controlled } \\
\text { trial }\end{array}$ & Mexico/30-60 & $\begin{array}{l}\text { Patients with Type } 2 \\
\text { Diabetes }\end{array}$ & 81 & $\mathrm{~W}$ and $\mathrm{M}$ & 3 months & $\begin{array}{l}\text { A reduced-energy diet } \\
\text { with a dietary portfolio } \\
\text { (DP) ( } 14 \mathrm{~g} \text { of } \\
\text { dehydrated nopal, } 4 \mathrm{~g} \\
\text { of chia seeds, } 30 \mathrm{~g} \text { of } \\
\text { soy protein and } 4 \mathrm{~g} \text { of } \\
\text { inulin) }\end{array}$ & $\begin{array}{l}\text { Placebo }(28 \mathrm{~g} \text { of calcium } \\
\text { caseinate and } 15 \mathrm{~g} \text { of } \\
\text { maltodextrin) }\end{array}$ & $\begin{array}{l}\text { DP consumption increased levels of } \\
\text { A. muciniphila by approximately } 125 \% \text {. }\end{array}$ \\
\hline $\begin{array}{l}\text { Pinheiro I } \\
\text { et al. } 2017 \text { [11] }\end{array}$ & $\begin{array}{l}\text { Randomized, } \\
\text { double-blind } \\
\text { placebo-controlled } \\
\text { trial }\end{array}$ & Belgium/20-69 & $\begin{array}{l}\text { Healthy with reduced } \\
\text { bowel movements } \\
\text { and other symptoms } \\
\text { of GI discomfort } \\
\text { stratified in severe } \\
\text { and moderate }\end{array}$ & 80 & $\mathrm{~W}$ and $\mathrm{M}$ & 6 weeks & $\begin{array}{l}\text { EpiCor fermentate } \\
(500 \mathrm{mg} / \mathrm{d})\end{array}$ & $\begin{array}{l}\text { Placebo (maltodextrin } \\
(500 \mathrm{mg} / \mathrm{d}))\end{array}$ & $\begin{array}{l}\text { Significant relative increase of } A \text {. } \\
\text { muciniphila in the moderate GI } \\
\text { discomfort symptoms group at visit } \\
\text { week } 3(p=0.0001) \text { and visit week } 6 \\
(p=0.036)\end{array}$ \\
\hline $\begin{array}{l}\text { Roshanravan N } \\
\text { et al. } 2017 \text { [25] }\end{array}$ & $\begin{array}{l}\text { Randomized, } \\
\text { double-blind } \\
\text { placebo-controlled } \\
\text { trial }\end{array}$ & Iran/30-55 & $\begin{array}{l}\text { Overweight and } \\
\text { obese diabetes } \\
\text { patients }\end{array}$ & 60 & $\mathrm{~W}$ and $\mathrm{M}$ & 6 weeks & $\begin{array}{l}\text { Group A: Butyrate } \\
\text { group }(600 \mathrm{mg} / \mathrm{d} \\
\text { sodium butyrate }+ \\
\text { inulin placebo) } \\
\text { Group B: inulin group } \\
(10 \mathrm{~g} / \mathrm{d} \text { inulin powder }+ \\
\text { butyrate placebo }) \\
\text { Group C: butyrate }+ \\
\text { inulin group }(600 \mathrm{mg} / \mathrm{d} \\
\text { sodium butyrate }+ \\
10 \mathrm{~g} / \mathrm{d} \text { inulin powder })\end{array}$ & $\begin{array}{l}\text { Butyrate }+ \text { inulin } \\
\text { placebo }(6 \\
\text { starch capsules } \\
(100 \mathrm{mg}) \text { and } 10 \mathrm{~g} \text { of } \\
\text { starch powder })\end{array}$ & $\begin{array}{l}\text { The percentage changes of } A \text {. } \\
\text { muciniphila abundance indicated a } \\
\text { significant increase in group taking } \\
\text { sodium butyrate and inulin (group A } \\
\text { and B) in comparison with the placebo } \\
\text { group }(p<0.05) \text {. A non-significant rise } \\
\text { in this bacterium concentration was } \\
\text { seen after supplementation with both } \\
\text { sodium butyrate and inulin (group C). }\end{array}$ \\
\hline $\begin{array}{l}\text { Walker JM et al. } \\
2019[27]\end{array}$ & $\begin{array}{l}\text { Randomized, } \\
\text { double-blind } \\
\text { placebo-controlled } \\
\text { trial }\end{array}$ & USA/30-70 & $\begin{array}{l}\text { Obese insulin } \\
\text { resistant subjects with } \\
\text { metabolic syndrome }\end{array}$ & $28^{\mathrm{a}}$ & $\mathrm{M}$ & 5 weeks & $\begin{array}{l}\text { Resveratrol ( } 500 \mathrm{mg} \\
\text { Mega-RES } 99 \% \\
\text { capsules twice daily) }\end{array}$ & $\begin{array}{l}\text { Placebo (two } 500 \mathrm{mg} \\
\text { placebo capsules twice } \\
\text { daily) }\end{array}$ & $\begin{array}{l}\text { Overall, there was no difference. } \\
\text { However, when split by ethnicity, } \\
\text { resveratrol administration to } \\
\text { Caucasian subjects led to an increase } \\
\text { in } A \text {. muciniphila compared to the } \\
\text { non-Caucasians. }\end{array}$ \\
\hline
\end{tabular}

* Studies examining both A. Muciniphila and F. Prausnitzii. FODMAPs Fermentable Oligosaccharides, Disaccharides, Monosaccharides and Polyols; FOS fructo-oligosaccharides;

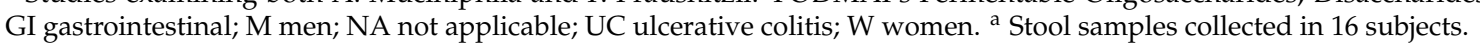


Table 2. Descriptive Summary of Randomized Clinical Trials Investigating the Associations Between Dietary Interventions and Faecalibacterium prausnitzii.

\begin{tabular}{|c|c|c|c|c|c|c|c|c|c|}
\hline \multirow{2}{*}{$\begin{array}{l}\text { Lead Author, } \\
\text { Publication Year }\end{array}$} & \multirow{2}{*}{ Study Design } & \multirow{2}{*}{$\begin{array}{l}\text { Location/Age } \\
\text { Range }\end{array}$} & \multirow{2}{*}{$\begin{array}{l}\text { Individual Health } \\
\text { Status }\end{array}$} & \multirow{2}{*}{$\begin{array}{l}\text { Total } \\
\text { Participants }\end{array}$} & \multirow{2}{*}{ Sex } & \multirow{2}{*}{$\begin{array}{l}\text { Period of } \\
\text { Intervention }\end{array}$} & \multicolumn{2}{|c|}{ Dietary Treatment Characteristics } & \multirow{2}{*}{ Main Findings } \\
\hline & & & & & & & Intervention Type & Control Type & \\
\hline $\begin{array}{l}\text { Benjamin JL et al. } \\
2011 \text { [12] }\end{array}$ & $\begin{array}{l}\text { Randomized } \\
\text { double-blinded } \\
\text { placebo-controlled } \\
\text { trial }\end{array}$ & $\mathrm{UK} / 39.5 \pm 14.4$ & $\begin{array}{l}\text { Patients with Crohn's } \\
\text { disease }\end{array}$ & 103 & M & 4 weeks & $\begin{array}{l}\text { Normal diet } \\
\text { supplemented with } \\
15 \mathrm{~g} / \text { day FOS, } \\
\text { comprising fructose } \\
\text { polymers of differing } \\
\text { chain lengths }\end{array}$ & $\begin{array}{l}\text { Placebo (maltodextrin } \\
15 \mathrm{~g} / \text { day) }\end{array}$ & $\begin{array}{l}\text { No significant differences between } \\
\text { patients in the FOS and placebo group at } \\
\text { week } 4(p=0.95) \text {. }\end{array}$ \\
\hline $\begin{array}{l}\text { Benus RFJ et al. } \\
2010 \text { [52] }\end{array}$ & $\begin{array}{l}\text { Randomized } \\
\text { double-blinded } \\
\text { cross-over trial }\end{array}$ & $\mathrm{UK} / 21-34$ & Healthy & 14 & $\mathrm{~W}$ and $\mathrm{M}$ & 4 weeks & $\begin{array}{l}\text { A formula } \\
\text { supplemented } \\
\text { with dietary fibre } \\
\text { (14 g/l) consisting of } \\
\text { pea fibre and } \\
\text { fructo-oligosaccharides } \\
\text { A fibre-free enteral } \\
\text { formula }\end{array}$ & NA & $\begin{array}{l}\text { There were large and statistically } \\
\text { significant reductions in the numbers of } \\
\text { the } F \text {. prausnitzzii group during both the } \\
\text { fibbe-free and fibre-supplemented diets. } \\
\text { No differences between the fibre-free } \\
\text { and fibre-supplemented diet }(p=0.23)\end{array}$ \\
\hline $\begin{array}{l}\text { Blatchford P et al. } \\
2017^{*}[1]\end{array}$ & $\begin{array}{l}\text { Randomized } \\
\text { double-blind } \\
\text { placebo-controlled } \\
\text { cross-over trial }\end{array}$ & $\begin{array}{l}\text { New } \\
\text { Zealand/23-56 }\end{array}$ & $\begin{array}{l}\text { Healthy participants who } \\
\text { had no clinical symptoms } \\
\text { of constipation and } \\
\text { functionally constipated } \\
\text { participants }\end{array}$ & $29 / \mathrm{W}$ and $\mathrm{M}$ & $\mathrm{W}$ and $\mathrm{M}$ & $\begin{array}{l}4 \text { weeks each } \\
\text { intervention }(2 \text { weeks } \\
\text { washout period } \\
\text { between each } \\
\text { intervention) }\end{array}$ & $\begin{array}{l}\text { ACTAZINTM } \mathrm{L} \\
(600 \mathrm{mg} / \mathrm{d}) \\
\text { ACTAZINTM } \mathrm{H} \\
(2400 \mathrm{mg} / \mathrm{d}) \\
\text { Livaux } \\
\text { Probiotic group: }\end{array}$ & $\begin{array}{l}\text { Placebo (isomalt } \\
\text { coloured green } \\
2400 \mathrm{mg} / \mathrm{d} \text { ) }\end{array}$ & $\begin{array}{l}\text { F. prausinitzii abundance significantly } \\
\text { increased from } 3.4 \text { to } 7.0 \% \text { following } \\
\text { Livaux } \mathrm{T}^{\mathrm{TM}} \text { supplementation in the } \\
\text { functionally constipated group } \\
(p=0.024) \text {. }\end{array}$ \\
\hline $\begin{array}{l}\text { Clavel T et al. } \\
2005[2]\end{array}$ & $\begin{array}{l}\text { Randomized } \\
\text { double-blind } \\
\text { placebo-controlled } \\
\text { trial }\end{array}$ & $\begin{array}{l}\text { France/ } \\
60.4 \pm 7.1\end{array}$ & Postmenopausal women & 39 & $\mathrm{~W}$ & 30 days & $\begin{array}{l}\text { isoflavones } \\
(100 \mathrm{mg} / \mathrm{d})+B \\
\text { animalis } \mathrm{DN}-173010 \\
\text { Prebiotic group: } \\
\text { isoflavones }(100 \\
\mathrm{mg} / \mathrm{d})+ \text { FOS }(7 \mathrm{~g} / \mathrm{d})\end{array}$ & $\begin{array}{l}\text { Placebo (isoflavones } \\
100 \mathrm{mg} / \mathrm{d} \text { ) }\end{array}$ & $\begin{array}{l}\text { Bacterial percentages for } F \text {. prausnitzii } \\
\text { subgroup decreased significantly in } \\
\text { control subjects compared to the } \\
\text { probiotic and prebiotic group }(p=0.034)\end{array}$ \\
\hline $\begin{array}{l}\text { Dewulf EM et al. } \\
2012[3]\end{array}$ & $\begin{array}{l}\text { Double-blind } \\
\text { placebo-controlled } \\
\text { trial }\end{array}$ & $\begin{array}{l}\text { Belgium/ } \\
47.5 \pm 8.5\end{array}$ & Obese & 30 & W & 3 months & $\begin{array}{l}\text { ITF prebiotics } \\
\text { (Synergy 1, namely, } \\
\text { inulin/oligofructose } \\
50 / 50 \text { mix) }\end{array}$ & Placebo (maltodextrin) & $\begin{array}{l}\text { Treatment with ITF prebiotics, but not } \\
\text { the placebo, led to an increase in } \\
\text { F. prausnitzii. }\end{array}$ \\
\hline $\begin{array}{l}\text { Fava F et al. } \\
2013[14]\end{array}$ & $\begin{array}{l}\text { Five-arm parallel, } \\
\text { placebo-controlled, } \\
\text { single-blind study }\end{array}$ & $\mathrm{UK} / 56.0 \pm 9.5$ & $\begin{array}{l}\text { Individuals at increased } \\
\text { risk of metabolic } \\
\text { syndrome }\end{array}$ & 88 & $\mathrm{~W}$ and $\mathrm{M}$ & 24 weeks & $\begin{array}{l}\text { High SFA diet } \\
\text { High MUFA/high GI } \\
\text { High MUFA/Low GI } \\
\text { High CHO/High GI } \\
\text { High CHO/Low GI }\end{array}$ & NA & $\begin{array}{l}\text { Numbers of } F \text {. prausnitzii increased after } \\
\text { intervention with high CHO and low GI } \\
(p=0.022) \text { and high SFA }(p=0.018) \text { diet } \\
\text { compared to baseline. }\end{array}$ \\
\hline $\begin{array}{l}\text { Fernando WMU } \\
\text { et al. } 2010[4]\end{array}$ & $\begin{array}{l}\text { Randomized } \\
\text { cross-over trial }\end{array}$ & $\begin{array}{l}\text { Canada/ } \\
25.6 \pm 8.7\end{array}$ & Healthy & 12 & $\mathrm{~W}$ and $\mathrm{M}$ & 9 weeks & $\begin{array}{l}\text { Control diet }+5 \mathrm{~g} / \mathrm{d} \\
\text { raffinose } \\
\text { Control diet }+200 \mathrm{~g} / \mathrm{d} \\
\text { canned chickpea }\end{array}$ & Control diet & $\begin{array}{l}\text { F. prausnitzii was more abundant in the } \\
\text { raffinose diet and the chickpea diet } \\
\text { compared to the control diet. }\end{array}$ \\
\hline $\begin{array}{l}\text { Guadamuro L } \\
\text { et al. } 2015[15]\end{array}$ & Single-arm trial & Spain/48-61 & $\begin{array}{l}\text { Menopausal women with } \\
\text { no chronic disease }\end{array}$ & 16 & $\mathrm{~W}$ & 24 weeks & $\begin{array}{l}\text { One tablet } \\
\text { isoflavoneconcentrate } \\
(80 \mathrm{mg}) \text { per day }\end{array}$ & NA & $\begin{array}{l}\text { There was an increase in the intensity of } \\
\text { F. prausnitzii. }\end{array}$ \\
\hline $\begin{array}{l}\text { Halmos EP et al. } \\
2015^{*}[16]\end{array}$ & $\begin{array}{l}\text { Single-arm blinded } \\
\text { randomized } \\
\text { cross-over trial }\end{array}$ & Australia/18+ & $\begin{array}{l}\text { Irritable bowel syndrome } \\
\text { and healthy individuals }\end{array}$ & 33 & $\mathrm{~W}$ and $\mathrm{M}$ & 6 weeks & $\begin{array}{l}\text { Diet low in } \\
\text { FODMAPs }\end{array}$ & $\begin{array}{l}\text { Diet containing } \\
\text { FODMAP content of a } \\
\text { typical Australian diet }\end{array}$ & $\begin{array}{l}\text { Low FODMAP diet reduced total } \\
\text { bacterial abundance, but did not impact } \\
\text { relative abundance of } F \text {. prausnitzii. }\end{array}$ \\
\hline $\begin{array}{l}\text { Halmos EP et al. } \\
2016^{*}[17]\end{array}$ & $\begin{array}{l}\text { Single-arm blinded } \\
\text { randomized } \\
\text { cross-over trial }\end{array}$ & Australia/18+ & $\begin{array}{l}\text { Patients with clinically } \\
\text { quiescent Crohn's disease }\end{array}$ & 9 & $\mathrm{~W}$ and $\mathrm{M}$ & 6 weeks & $\begin{array}{l}\text { Diet low in } \\
\text { FODMAPs }\end{array}$ & $\begin{array}{l}\text { Diet containing } \\
\text { FODMAP content of a } \\
\text { typical Australian diet }\end{array}$ & $\begin{array}{l}\text { No significant difference in F. prausnitzii } \\
\text { between the two diets. }\end{array}$ \\
\hline
\end{tabular}


Table 2. Cont

\begin{tabular}{|c|c|c|c|c|c|c|c|c|c|}
\hline \multirow{2}{*}{$\begin{array}{l}\text { Lead Author, } \\
\text { Publication Year }\end{array}$} & \multirow{2}{*}{ Study Design } & \multirow{2}{*}{$\begin{array}{l}\text { Location/Age } \\
\text { Range }\end{array}$} & \multirow{2}{*}{$\begin{array}{l}\text { Individual Health } \\
\text { Status }\end{array}$} & \multirow{2}{*}{$\begin{array}{l}\text { Total } \\
\text { Participants }\end{array}$} & \multirow{2}{*}{ Sex } & \multirow{2}{*}{$\begin{array}{l}\text { Period of } \\
\text { Intervention }\end{array}$} & \multicolumn{2}{|c|}{ Dietary Treatment Characteristics } & \multirow{2}{*}{ Main Findings } \\
\hline & & & & & & & Intervention Type & Control Type & \\
\hline $\begin{array}{l}\text { Hooda S et al. } \\
2012^{*}[6]\end{array}$ & $\begin{array}{l}\text { Randomized } \\
\text { double-blind } \\
\text { placebo-controlled } \\
\text { cross-over trial }\end{array}$ & $\begin{array}{l}\text { USA/ } \\
27.5 \pm 4.33\end{array}$ & Healthy & 25 & M & 9 weeks & $\begin{array}{l}\text { Polydextrose (PDX) } \\
\text { ( } 7 \mathrm{~g}, 3 \text { times per day) } \\
\text { Soluble corn fiber } \\
\text { (SCF) }(7 \mathrm{~g}, 3 \text { times per } \\
\text { day) }\end{array}$ & $\begin{array}{l}\text { Placebo: no } \\
\text { supplemental fiber } \\
\text { control (NFC) }(0 \mathrm{~g} \text {, } \\
3 \text { times per day) }\end{array}$ & $\begin{array}{l}\text { F. prausnitzii was greater in participants } \\
\text { when they consumed PDX or SCF than } \\
\text { when they consumed NFC }(p<0.05) \text {. }\end{array}$ \\
\hline $\begin{array}{l}\text { Hustoft TN et al. } \\
2016[7]\end{array}$ & $\begin{array}{l}\text { Randomized } \\
\text { double-blind } \\
\text { placebo-controlled } \\
\text { cross-over trial }\end{array}$ & Norway/18-52 & $\begin{array}{l}\text { Diarrhea-predominant or } \\
\text { mixed irritated bowel } \\
\text { syndrome }\end{array}$ & 20 & $\mathrm{~W}$ and $\mathrm{M}$ & $\begin{array}{l}10 \text { days each } \\
\text { intervention ( } 3 \text { weeks } \\
\text { washout period) }\end{array}$ & $\begin{array}{l}\text { Fructo-oligosaccharides } \\
\text { (FOS) } 16 \mathrm{~g} / \mathrm{d}\end{array}$ & $\begin{array}{l}\text { Placebo: Maltodextrin } \\
16 \mathrm{~g} / \mathrm{d}\end{array}$ & $\begin{array}{l}\text { Ten days of FOS supplementation } \\
\text { increased the level of F. prausnitzii. }\end{array}$ \\
\hline $\begin{array}{l}\text { James SL et al. } \\
2015^{*}[54]\end{array}$ & $\begin{array}{l}\text { Randomized } \\
\text { single-blind } \\
\text { cross-over trial }\end{array}$ & Australia/18-72 & $\begin{array}{l}\text { Patients with UC in } \\
\text { remission and healthy } \\
\text { subjects }\end{array}$ & 29 & $\mathrm{~W}$ and $\mathrm{M}$ & 8 weeks & $\begin{array}{l}\text { 'Low resistant starch } \\
(\mathrm{RS}) / \text { wheat bran }(\mathrm{WB}) \\
\text { foods containing } \\
2-5 \mathrm{~g} \text { RS and } \\
2-5 \mathrm{~g} \text { WB fibre per } \\
\text { day } \\
\text { 'High RS/WB' foods } \\
\text { containing } 15 \mathrm{~g} \\
\text { RS and } 12 \mathrm{~g} \mathrm{WB} \text { fibre } \\
\text { per day }\end{array}$ & NA & $\begin{array}{l}\text { For both cohorts, increasing the intake } \\
\text { of RS/WB gave no indication of changes } \\
\text { in relative or absolute abundance in } F \text {. } \\
\text { prausnitzii. }\end{array}$ \\
\hline $\begin{array}{l}\text { Lee T et al. } \\
2017 \text { [19] }\end{array}$ & $\begin{array}{l}\text { Randomized, } \\
\text { double-blind } \\
\text { placebo-controlled } \\
\text { trial }\end{array}$ & Canada/18+ & $\begin{array}{l}\text { Iron deficient } \\
\text { Inflammatory bowel } \\
\text { disease patients }\end{array}$ & 72 & $\mathrm{~W}$ and $\mathrm{M}$ & 12 weeks & $\begin{array}{l}\text { Oral iron sulfate } \\
300 \mathrm{mg} \text {, tablet, twice a } \\
\text { day }\end{array}$ & $\begin{array}{l}\text { Iron sucrose, } 300 \mathrm{mg} \text {, } \\
\text { intravenous, three or } \\
\text { four/day }\end{array}$ & $\begin{array}{l}\text { Lower abundance of } F \text {. prausnitzii after } \\
\text { oral iron therapy compared to } \\
\text { intravenous iron therapy }(p=0.009) \text {. }\end{array}$ \\
\hline $\begin{array}{l}\text { Majid HA et al. } \\
2014[8]\end{array}$ & $\begin{array}{l}\text { Multi-centre, } \\
\text { randomized } \\
\text { double-blind } \\
\text { controlled trial }\end{array}$ & $\mathrm{UK} / 70.8 \pm 9.7$ & $\begin{array}{l}\text { Patients from the ICU } \\
\text { starting exclusive } \\
\text { nasogastric enteral } \\
\text { nutrition }\end{array}$ & 22 & $\mathrm{~W}$ and $\mathrm{M}$ & Up to 14 days & $\begin{array}{l}\text { Oligofructose/inulin } \\
7 \mathrm{~g} / \mathrm{d}\end{array}$ & $\begin{array}{l}\text { Placebo: maltodextrin } \\
7 \mathrm{~g} / \mathrm{d}\end{array}$ & $\begin{array}{l}\text { There were significantly lower } \\
\text { concentrations of } F \text {. prausnitzii in } \\
\text { patients receiving additional } \\
\text { oligofructose/inulin }(p=0.01) \text {. }\end{array}$ \\
\hline $\begin{array}{l}\text { Medina-Vera I } \\
\text { et al. } 2019^{*}[9]\end{array}$ & $\begin{array}{l}\text { Single-centre } \\
\text { randomized, } \\
\text { controlled, } \\
\text { double-blind } \\
\text { parallel-group trial }\end{array}$ & Mexico/30-60 & $\begin{array}{l}\text { Patients with Type } 2 \\
\text { Diabetes }\end{array}$ & 81 & $\mathrm{~W}$ and $\mathrm{M}$ & 3 months & $\begin{array}{l}\text { A reduced-energy } \\
\text { diet with a dietary } \\
\text { portfolio (DP) } \\
\text { comprising } 14 \mathrm{~g} \text { of } \\
\text { dehydrated nopal, } 4 \mathrm{~g} \\
\text { of chia seeds, } 30 \mathrm{~g} \text { of } \\
\text { soy protein and } 4 \mathrm{~g} \text { of } \\
\text { inulin }\end{array}$ & $\begin{array}{l}\text { Placebo, comprising of } \\
28 \mathrm{~g} \text { of calcium } \\
\text { caseinate and } 15 \mathrm{~g} \text { of } \\
\text { maltodextrin. }\end{array}$ & $\begin{array}{l}\text { Dietary intervention with functional } \\
\text { foods significantly modified faecal } \\
\text { microbiota compared with placebo. DP } \\
\text { consumption for } 12 \text { weeks increased } \\
\text { levels of F. prausnitzii by approximately } \\
34 \% \text {. }\end{array}$ \\
\hline $\begin{array}{l}\text { Moreno-Indias I } \\
\text { et al. } 2016[21]\end{array}$ & $\begin{array}{l}\text { Randomized, } \\
\text { cross-over } \\
\text { controlled trial }\end{array}$ & Spain/45-50 & $\begin{array}{l}\text { Metabolic syndrome and } \\
\text { healthy individuals }\end{array}$ & 20 & M & 10 weeks ( 75 days) & $\begin{array}{l}\text { Red wine, } 272 \\
\mathrm{~mL} / \text { day }\end{array}$ & $\begin{array}{l}\text { De-alcoholized (no } \\
\text { ethanol) red wine, } \\
272 \mathrm{~mL} / \text { dat }\end{array}$ & $\begin{array}{l}\text { In metabolic syndrome patients, there } \\
\text { was a significant increase of } F \text {. } \\
\text { prausnitziii, after the red wine and } \\
\text { de-alcoholized red wine intake periods } \\
\text { compared to baseline. In the healthy } \\
\text { group, a significant increase in the } \\
\text { number of } F \text {. prausnitzii through the } \\
\text { intervention period was observed. }\end{array}$ \\
\hline $\begin{array}{l}\text { Most J et al. } \\
2017[10]\end{array}$ & $\begin{array}{l}\text { Randomized } \\
\text { double-blind } \\
\text { placebo-controlled } \\
\text { trial }\end{array}$ & $\begin{array}{l}\text { The } \\
\text { Netherlands/ } \\
20-50\end{array}$ & Obese & 42 & $\mathrm{~W}$ and $\mathrm{M}$ & 12 weeks & $\begin{array}{l}\text { A combination of } \\
\text { epigallocatechin-3-gallat } \\
\text { (EGCG) and } \\
\text { resveratrol (RES) } \\
\text { supplements (EGCG } \\
+ \text { RES; } 282 \text { and } 80 \\
\text { mg/day, respectively) }\end{array}$ & $\begin{array}{l}\text { tePlacebo (partly } \\
\text { hydrolyzed } \\
\text { microcrystalline } \\
\text { cellulose-filled } \\
\text { supplements) }\end{array}$ & $\begin{array}{l}\text { EGCG+RES supplementation } \\
\text { significantly decreased Bacteroidetes } \\
\text { and tended to reduce } F \text {. prausnitzii in } \\
\text { men }(p=0.05 \text { and } p=0.10 \text {, respectively) } \\
\text { but not in women }(P=0.15 \text { and } P=0.77 \text {, } \\
\text { respectively). }\end{array}$ \\
\hline
\end{tabular}


Table 2. Cont.

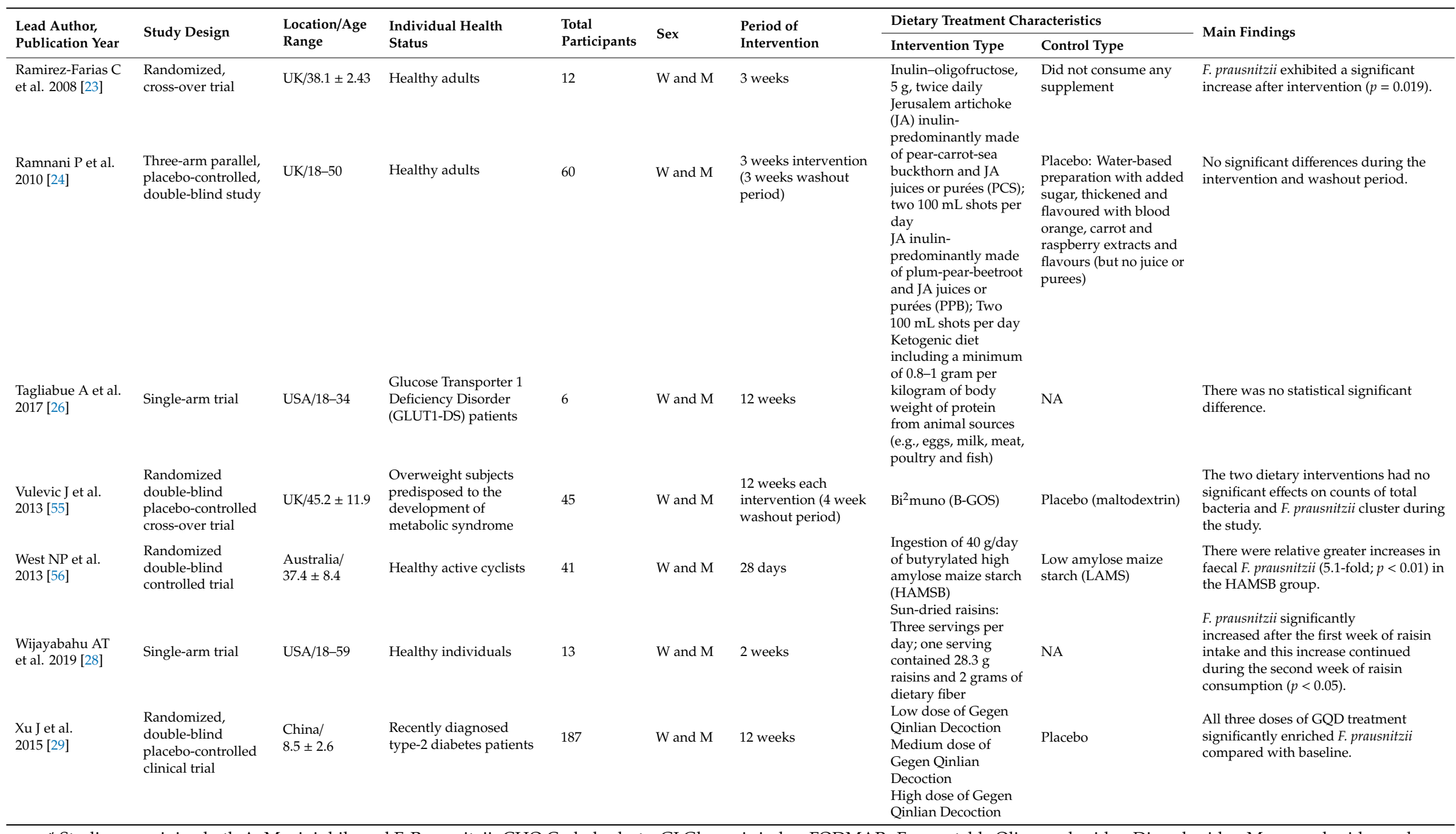

* Studies examining both A. Muciniphila and F. Prausnitzii. CHO Carbohydrate; GI Glycemic index; FODMAPs Fermentable Oligosaccharides, Disaccharides, Monosaccharides and Polyols; FOS fructo-oligosaccharides; ICU intensive care unit; ITF Inulin-type fructans; M men; MUFA monosaturated fat; SFA Saturated fatty acids; UC ulcerative colitis; W women. 
Treatment with prebiotic inulin-type fructans led to an increase of $F$. prausnitzii compared to placebo (maltodextrin) in obese women [36]. Supplementation of fructo-oligosaccharides, which are prebiotic fructans, increased the level of F. prausnitzii compared to placebo (maltodextrin) in patients with diarrhoea or mixed irritated bowel syndrome [41]. Supplementation of prebiotic inulin-oligofructose also increased the level of $F$. prausnitzii in healthy individuals [26]. Moreno-Indias I. et al. studied the prebiotic effect of red wine in male metabolic syndrome patients and healthy individuals and reported an increase of F. prausnitzii for red wine intake compared to baseline [46]. Another study found that F. prausnitzii was greater when healthy men consumed polydextrose or soluble corn fiber supplementation, which could be potential prebiotics, than when they consumed no supplemental fiber [40]. Medina-Vera I. et al. examined the effect of a reduced energy diet with prebiotic properties compared to a placebo diet in patients with type 2 diabetes, and reported an increase of $F$. prausnitzii of $34 \%$ [45]. Fernando WMU et al. studied the prebiotic potential of chickpea oligosaccharides (raffinose) alone or as components of chickpea and found that $F$. prausnitzii was more abundant in the raffinose diet and the chickpea diet compared to the control diet [38]. Ingestion of butyrylated high amylose maize starch led to greater relative increases of $F$. prausnitzii compared to ingestion of low amylose maize starch [55].

However, there are also studies reporting a decrease in F. prausnitzii after a prebiotic intervention. Patients from the intensive care unit starting nasogastric enteral nutrition receiving additional oligofructose/inulin had significantly lower concentration of $F$. prausnitzii compared to patients receiving placebo [44]. A combination of epigallocatechin-3-gallate and resveratrol decreased F. prausnitzii compared to placebo in obese men but not in obese women [47]. A cross-over trial comparing a fibre-free enteral formula or a formula supplemented with dietary fibre consisting of pea fibre and fructo-oligosaccharides showed large reductions in the number of F. prausnitzii during both the fibre-free and fibre-supplemented diets among healthy participants [52].

Moreover, some studies reported no differences in F. prausnitzii after a prebiotic intervention. Halmos E. et al. examined a diet low in FODMAPs compared to a diet containing FODMAPs in two different studies in patients with irritated bowel syndrome and patients with clinically quiescent Crohn's disease $[24,25]$. They did not report significant differences in F. prausnitzii between the diets in both studies. Another study conducted among men with Crohn's disease examining the effect of fructo-oligosaccharides compared to a non-prebiotic carbohydrate also did not found differences in F. prausnitzii [32]. Vulevic J. et al. investigated the effect of administering Bi2muno (B-GOS) compared to placebo (maltodextrin) in a cross-over trial among overweight subjects predisposed to the development of metabolic syndrome, and reported no significant effects on counts of the F. prausnitzii cluster during the study [54]. Increasing the intake of resistant starch and wheat bran foods containing fibres did not change the relative and absolute abundance of $F$. prausnitzii in healthy participants and patients with ulcerative colitis in remission [53]. Among healthy individuals, Ramnani P. et al. studied the prebiotic effect of Jerusalem artichoke compared to a placebo, but did not report significant differences in F. prausnitzii [27].

Clavel T. et al. studied the effect of a prebiotic (isoflavones and fructo-oligosaccharides) or probiotic (isoflavones and B. animalis DN-173 010) compared to placebo (isoflavones alone) in postmenopausal women [34]. They reported that percentages of $F$. prausnitzii decreased significantly in control subjects compared to probiotic and prebiotic groups. Guadamuro L. et al. examined the effect of isoflavone concentrate supplementation alone in menopausal women and found an increase in the intensity of F. prausnitzii after isoflavone supplementation [39].

Fava F. et al. showed that the type and quantity of dietary fat and carbohydrate can alter faecal microbiome in individuals at increased risk of metabolic syndrome, whereby F. prausnitzii increased after intervention of a diet with high monounsaturated fatty acids and a high glycaemic index, compared to diets with high saturated fatty acids, high monounsaturated fatty acids in combination with a low glycaemic index, or with high carbohydrates in combination with high or low glycaemic index [37]. A ketogenic diet including a minimum of 0.8-1 gram per kilogram of bodyweight of 
protein from animal sources did not have an effect on F. prausnitzii [49]. Consumption of kiwifruit capsules increased F. prausnitzii abundance in functionally constipated individuals, compared to placebo [33]. Addition of sun-dried raisins to the diet also increased the abundance of $F$. prausnitzii among healthy individuals [50]. Oral iron therapy resulted in lower abundance of $F$. prausnitzii compared to intravenous iron therapy among iron deficient inflammatory bowel disease patients [42]. $\mathrm{Xu} \mathrm{J}$ et al. examined the effect of a Chinese herbal formula (Gegen Qinlian Decoction) in a low, medium and high dose in recently diagnosed type 2 diabetes patients. They reported that all three doses of Gegen Qinlian Decoction treatment significantly enriched F. prausnitzii compared with baseline [51].

\section{Discussion}

In the current systematic review of clinical trials, diet-induced changes in A. muciniphila and F. prausnitzii were reviewed. An increase in abundance of $A$. muciniphila was observed after a caloric restriction diet, supplementation with pomegranate extract, resveratrol, polydextrose, EpiCor or sodium butyrate, whereas a diet low in FODMAPs decreased the abundance of A. muciniphila. Prebiotics use was the main dietary intervention investigated in relation to F. prausnitzii, showing contradictory results depending on the type of prebiotics. Isoflavone supplementation and intake of some types of fatty acids, such as monounsaturated fatty acids, was associated with increased abundance of $F$. prausnitzii, whereas inulin intake and a reduced energy diet increased the abundance of both A. muciniphila and F. prausnitzii.

Approximately 100 trillion microorganisms are present in the body but mostly residing in the gastrointestinal tract [56]. In response to environmental changes, the gut microbiome can affect gene expression in humans. While around 23,000 genes make up the human genome, the microbiome consists over 3 million genes producing thousands of metabolites, and therefore affecting many aspects of human health, including gastrointestinal and cardiometabolic phenotypes [56,57]. Changes in microbiota are observed in patients with inflammatory gastrointestinal disease, prediabetes, and T2D, particularly a decreased abundance of $A$. muciniphila and F. prausnitzii [58]. In addition, low abundance of $A$. muciniphila and F. prausnitzii has been associated with increased inflammatory processes and atherosclerosis, and F. prausnitzii transplantation has been shown to be an effective therapeutic approach for diabetes and its complications [59]. F. prausnitzii is an important butyrate-producer with anti-inflammatory properties, while $A$. muciniphila degrades mucin in the gut lining resulting in syntrophic interactions and stimulation of intestinal metabolite pool [60]. For example, co-cultivations of A. muciniphila with butyrate-producing bacteria resulted in syntrophic growth and butyrate production [60]. Butyrate plays an important role in anti-inflammation and maintaining intestinal barrier integrity by modulating intestinal macrophages' function, downregulating lipopolysaccharide-induced pro-inflammatory mediators, such as nitric oxide, IL-6, and IL-12, inducing the differentiation of regulatory $\mathrm{T}$ cells and stabilizing hypoxia-inducible factor [61-64]. Further, butyrate can have beneficial effects on glucose and energy homeostasis by activating intestinal gluconeogenesis, as well as inducing apoptosis of colon cancer cells [65]. Studies in animal models have shown that colonization by A. muciniphila resulted in transcriptional changes, leading to an increase in the expression of genes associated with immune responses and cellular lipid metabolism [11,66]. Besides degrading mucins, A. muciniphila can also stimulate mucin production, suggesting an autocatalytic process $[67,68]$. Mucins are large, highly glycosylated proteins that play a crucial role in luminal protection of the gastrointestinal tract, thereby reducing translocation of pro-inflammatory lipopolysaccharides, controlling fat storage, adipose tissue metabolism, and glucose homeostasis [68,69]. Therefore, altering the abundance and dynamics of these two strains of bacteria can lead to alterations in metabolic processes, and prevention and management of metabolic diseases.

Diet is the main source of energy to humans, but can also modulate microbiota and impact host-microbe interactions [70]. Therefore, gut microbiota may be crucial in mediating the health effects of foods. The gut microbiota provides the exoenzymes to catalyze and ferment substrates that cannot be completely digested by the human digestive tract to produce metabolites such as short 
fatty acids including butyrate [71]. Such non-digestible substrates include complex carbohydrates derived from plants and dietary fibres from cereals, legumes, vegetables, fruits, and nuts. Modulation of the gut microbiome through adherence to a high-fibre plant-based diet has been suggested as a potential therapeutic approach for prevention and treatment of inflammatory gastrointestinal diseases and metabolic diseases such as T2D and restoration of microbiota function [72,73]. In line with this hypothesis, we found in the current review that inulin-type fructans, fructo-oligosaccharides, polydextrose or soluble corn fiber supplementation, and raffinose can lead to increase abundance of A. muciniphila and/or F. prausnitzii. However, we found no consistent associations or decreases for other types of prebiotics and abundance of $F$. prausnitzii such as inulin-oligofructose and resistant starch or wheat bran foods. Yet, this inconsistency is somehow expected considering that studies used different types of prebiotics, for which bacteria have differing specificity and therefore affecting differently the number of fermentable substrates in the gut [14]. In addition, different types of fibres have differing effects in the luminal $\mathrm{pH}$ and transit rate. Type of fibre being consumed, gut transit time, and the functional capabilities of gut microbiota influences the fermentation of carbohydrate and subsequent regional delivery of metabolites [14]. Hence, the different results we see in this study on the association between prebiotics and F. prausnitzii may reflect this complexity. This is also in line with the findings from observational studies showing that some types of dietary fibres (such as those from cereals) but not all are associated with reduced risk of T2D [74].

Pomegranate extract, a rich and varied source of polyphenolic compounds, and resveratrol polyphenol have been widely investigated for their antioxidant, anti-inflammatory, anti-diabetic and anti-atherogenic properties in both genders [75,76]. Phytoestrogens seem to have similar effects but mainly in women; use of phytoestrogens in women is associated with improvement of menopausal symptoms, better glycaemic control and reduced risk of T2D [77,78]. However, the mechanisms of action of these compounds remain unclear. Microbiome plays an important role in metabolizing the non-absorbed fraction of these compounds, and therefore defining the ability of these compounds such as polyphenols to exert health effects [79]. On the other hand, pomegranate and polyphenol resveratrol can modulate the abundance of $A$. muciniphila, while isoflavone phytoestrogens alone, and not in combination with fibres, can modulate the abundance of $F$. prausnitzii $[19,23,34,39,43]$. While it is not completely clear how such compounds can affect intestinal microorganisms, limited evidence suggests that it could be either by the selective pressure they exert on specific microorganisms, or by modifying environmental conditions of the intestinal tract [34].

Modifying the diet can lead to rapid changes in microbiota within the first days. A study reported human gut microbiome changes after only 24 hours after shifting between plant and animal protein-based diets [80]. Similarly, the studies included in this review showed that dietary interventions are associated with changes in A. muciniphila and F. prausnitzii in the first weeks of intervention $[26,41,44,50]$. This suggests that diet may be an important and fast modifier of $A$. muciniphila and F. prausnitzii, and the microbiome in general, and a promising future therapeutic approach to prevent and treat inflammatory and metabolic conditions associated with specific microbiome strains. Our study highlights several additional factors that might be important to consider for a more comprehensive understanding of diet-related diseases and to elucidate the links between nutrition and dynamics of A. muciniphila and F. prausnitzii. However, dietary effects on microbiome may depend on health status, the baseline abundance of bacteria strains already in the gut, ethnicity, medication, and sex $[24,25,32-35,37,41,42,45,46,51]$. This could also explain the inconsistent results across studies in this systematic review, as we included studies among different sexes, ethnicities and health statuses. For instance, men and women have different genetic background, energy and nutritional requirements across the lifespan, as well as differences in gastrointestinal transit time, which can contribute to sex differences in microbiome [81,82]. Therefore, considering the sex-differences in the gut microbiome can provide novel insights in tailoring interventions and treatment, and therefore improving precision medicine. Machine-learning algorithms can help to predict inflammatory metabolic responses to meals, microbiome and how that differs by sex and ethnicity, and thus help to improve the emerging concept 
of personalized nutrition. For example, Zeevi et al. [83] used a machine-learning algorithm to predict personalised glucose responses after meals based on clinical and gut microbiome data. Based on the algorithm, a dietary intervention was designed and successfully shown to normalize blood glucose levels in a double-blinded randomised crossover trial of 26 patients [83]. Future research based on a large population-based studies and clinical trials is needed to evaluate a greater variety of food components and establish whether such personalised nutritional approaches based on microbiome composition are feasible and can improve clinical outcomes.

Further, it may be important to understand the differences that whole foods and ultra-processed foods may have on gut microbiota and subsequently on health outcomes. It has been shown that fibre-rich ultra-processed foods change the microbiota towards a less diverse and less beneficial composition compared to fibre-rich whole foods [84]. Processed foods have been undergone high heat and pressure, which can lead to several chemical and physical changes, including inactivation of endogenous enzymes, increased content of soluble dietary fibre and mechanical damage to the cell walls [85]. This suggests that whole unprocessed foods will enter the colon in an intact state which could favour the growth of bacteria that degrade fibre and produce beneficial metabolites, and thus exerting protective effects on health outcomes [85]. This theory is also supported from epidemiological studies showing that whole foods such as whole fish and whole grains can have a more beneficial effect than processed fish and refined grains, and that processed meat is in general associated with a stronger adverse health effect than whole meat [86]. Therefore, investigating the health effects of nutrients present in ultra-processed and whole food in the context of the microbiome can shed light on the mechanisms of the adverse effects of the Western diet.

To our knowledge, this is the first systematic review on the subject that critically and systematically appraised the literature on the effect of dietary interventions on A. muciniphila and F. prausnitzii. Nevertheless, some limitations from the included studies in this review merit careful consideration. Firstly, not all studies reported baseline and/or end of trial estimates between groups, and therefore the interpretation and the strength of the reported associations remained unclear. This and other factors (such as different types of study populations, interventions, and study designs) also limited providing a meaningful quantitative pooling of the existing data. It is important that future studies report summary estimates for all bacteria strains under investigation, and not apply selective reporting. Further, the studies included in this review were of suboptimal quality and the conclusions should be interpreted with caution. Most studies have been published in recent years and were conducted in limited sample sizes (16 studies had $\leq 30$ participants) within interventions lasting short time periods (15 studies lasted for $\leq 6$ weeks). Moreover, we cannot exclude the possibility that the changes we observed in A. muciniphila and F. prausnitzii are due to changes that dietary factors can lead to in the health condition of the participants, rather than the effect of diet alone. Nevertheless, most of clinical trials included in this review were performed in healthy individuals, and therefore we would expect that it is less likely that dietary factors we examined alter the abundances of the bacteria strains through changes in health. We also cannot exclude the possibility that the dietary factors lead to changes in other bacterial strains, which might have an effect on A. muciniphila and F. prausnitzii. What appears to be a promising field for intervention to improve health is also highly complex and new studies are required to untangle these complex interactions of diet, microbiome, and diseases in larger populations.

\section{Conclusions}

In summary, we found that specific dietary interventions could influence the abundance of A. muciniphila and F. prausnitzii, highlighting a novel potential mechanism describing how diet can affect inflammatory and metabolic health outcomes. Understanding the links between different types of dietary interventions and changes in abundance of $A$. muciniphila and $F$. prausnitzii can help shaping future nutritional recommendations and designing medical nutrition therapies that can help prevent and treat diseases related to these two strains of bacteria such as inflammatory gastrointestinal disease and T2D. 
Supplementary Materials: The following are available online at http://www.mdpi.com/2072-6643/11/7/1565/s1, Table S1: PRISMA 2009 checklist, Table S2: MOOSE checklist, Table S3: The Cochrane Collaboration's tool for assessing risk of bias.

Author Contributions: O.H.F. and T.M. designed and conceived the study. S.V., P.E.T., Z.M.R.D., M.G. and T.M. screened title/abstract, obtained full text, determined eligibility of articles and participated in data extraction. S.V., P.E.T., Z.M.R.D., P.M.-V., J.P.T., L.B., M.G., O.H.F. and T.M. participated in data synthesis/analysis and interpretation of the data. SV and TM drafted the final manuscript. S.V. and T.M. had full access to all of the data in the study and take responsibility for the integrity of the data and the accuracy of the data analysis. All authors contributed to the critical revision of the manuscript and approved the final version.

Funding: O.H.F. reported receiving grants or research support from Metagenics Inc. T.M. reported receiving personal fees from Standard Process.

Conflicts of Interest: The authors declare no conflict of interest.

\section{Appendix A}

Search Strategy

PUBMED 384

(akkermansia[All Fields] AND muciniphila[All Fields]) OR ("faecalibacterium prausnitzii"[MeSH Terms] OR ("faecalibacterium"[All Fields] AND "prausnitzii"[All Fields]) OR "faecalibacterium prausnitzii"[All Fields]) AND “humans"[MeSH Terms].

\section{EMBASE 1049}

(('akkermansia muciniphila*': ab, ti OR 'faecalibacterium prausnitzii*': ab, ti) OR ('akkermansia muciniphila'/exp OR ‘faecalibacterium prausnitzii'/exp)) AND ‘human'/de.

\section{References}

1. Cho, I.; Blaser, M.J. The human microbiome: At the interface of health and disease. Nat. Rev. Genet. 2012, 13, 260. [CrossRef] [PubMed]

2. Cao, Y.; Shen, J.; Ran, Z.H. Association between Faecalibacterium prausnitzii Reduction and Inflammatory Bowel Disease: A Meta-Analysis and Systematic Review of the Literature. Gastroenterol. Res. Pract. 2014, 2014, 872725. [CrossRef] [PubMed]

3. Davies, N.K.; O'Sullivan, J.M.; Plank, L.D.; Murphy, R. Altered gut microbiome after bariatric surgery and its association with metabolic benefits: A systematic review. Surg. Obes. Relat. Dis. Off. J. Am. Soc. Bariatr. Surg. 2019, 15, 656-665. [CrossRef] [PubMed]

4. Ferreira-Halder, C.V.; Faria, A.V.S.; Andrade, S.S. Action and function of Faecalibacterium prausnitzii in health and disease. Best Pract. Res. Clin. Gastroenterol. 2017, 31, 643-648. [CrossRef] [PubMed]

5. Geerlings, S.Y.; Kostopoulos, I.; de Vos, W.M.; Belzer, C. Akkermansia muciniphila in the Human Gastrointestinal Tract: When, Where, and How? Microorganisms 2018, 6, 75. [CrossRef]

6. Lopez-Siles, M.; Martinez-Medina, M.; Surís-Valls, R.; Aldeguer, X.; Sabat-Mir, M.; Duncan, S.H.; Flint, H.J.; Garcia-Gil, L.J. Changes in the Abundance of Faecalibacterium prausnitzii Phylogroups I and II in the Intestinal Mucosa of Inflammatory Bowel Disease and Patients with Colorectal Cancer. Inflamm. Bowel Dis. 2016, 22, 28-41. [CrossRef] [PubMed]

7. Gao, Z.; Yin, J.; Zhang, J.; Ward, R.E.; Martin, R.J.; Lefevre, M.; Cefalu, W.T.; Ye, J. Butyrate improves insulin sensitivity and increases energy expenditure in mice. Diabetes 2009, 58, 1509-1517. [CrossRef]

8. de Goffau, M.C.; Luopajarvi, K.; Knip, M.; Ilonen, J.; Ruohtula, T.; Härkönen, T.; Orivuori, L.; Hakala, S.; Welling, G.W.; Harmsen, H.J.; et al. Fecal microbiota composition differs between children with beta-cell autoimmunity and those without. Diabetes 2013, 62, 1238-1244. [CrossRef]

9. Li, J.; Lin, S.; Vanhoutte, P.P.; Woo, C.C.; Xu, A. Akkermansia Muciniphila Protects Against Atherosclerosis by Preventing Metabolic Endotoxemia-Induced Inflammation in Apoe-/- Mice. Circulation 2016, 133, 2434-2446. [CrossRef] 
10. Derrien, M.; Vaughan, E.E.; Plugge, C.C.; de Vos, W.M. Akkermansia muciniphila gen. nov., sp. nov., a human intestinal mucin-degrading bacterium. Int. J. Syst. Evol. Microbiol. 2004, 54 Pt 5, 1469-1476. [CrossRef]

11. Derrien, M.; Van Baarlen, P.; Hooiveld, G.; Norin, E.; Muller, M.; de Vos, W.M. Modulation of Mucosal Immune Response, Tolerance, and Proliferation in Mice Colonized by the Mucin-Degrader Akkermansia muciniphila. Front. Microbiol. 2011, 2, 166. [CrossRef]

12. Arumugam, M.; Raes, J.; Pelletier, E.; Le Paslier, D.; Yamada, T.; Mende, D.R.; Fernandes, G.R.; Tap, J.; Bruls, T.; Batto, J.M.; et al. Enterotypes of the human gut microbiome. Nature 2011, 473, 174-180. [CrossRef]

13. Louis, P.; Young, P.; Holtrop, G.; Flint, H.H. Diversity of human colonic butyrate-producing bacteria revealed by analysis of the butyryl-CoA:acetate CoA-transferase gene. Environ. Microbiol 2010, 12, 304-314. [CrossRef]

14. Ganesan, K.; Chung, S.S.; Vanamala, J.; Xu, B. Causal Relationship between Diet-Induced Gut Microbiota Changes and Diabetes: A Novel Strategy to Transplant Faecalibacterium prausnitzii in Preventing Diabetes. Int. J. Mol. Sci. 2018, 19, 3720. [CrossRef]

15. Schneeberger, M.; Everard, A.; Gomez-Valades, A.G.; Matamoros, S.; Ramírez, S.; Delzenne, N.M.; Gomis, R.; Claret, M.; Cani, P.D. Akkermansia muciniphila inversely correlates with the onset of inflammation, altered adipose tissue metabolism and metabolic disorders during obesity in mice. Sci. Rep. 2015, 5, 16643. [CrossRef]

16. Sokol, H.; Pigneur, B.; Watterlot, L.; Lakhdari, O.; Bermúdez-Humarán, L.G.; Gratadoux, J.J.; Blugeon, S.; Bridonneau, C.; Furet, J.P.; Corthier, G.; et al. Faecalibacterium prausnitzii is an anti-inflammatory commensal bacterium identified by gut microbiota analysis of Crohn disease patients. Proc. Natl. Acad. Sci. USA 2008, 105, 16731-16736. [CrossRef]

17. De Filippo, C.; Cavalieri, D.; Di Paola, M.; Ramazzotti, M.; Poullet, J.B.; Massart, S.; Collini, S.; Pieraccini, G.; Lionetti, P. Impact of diet in shaping gut microbiota revealed by a comparative study in children from Europe and rural Africa. Proc. Natl. Acad. Sci. USA 2010, 107, 14691-14696. [CrossRef]

18. Ejtahed, H.H.; Soroush, A.A.; Siadat, S.S.; Hoseini-Tavassol, Z.; Larijani, B.; Hasani-Ranjbar, S. Targeting obesity management through gut microbiota modulation by herbal products: A systematic review. Complement. Ther. Med. 2019, 42, 184-204. [CrossRef]

19. Anhe, F.F.; Pilon, G.; Roy, D.; Desjardins, Y.; Levy, E.; Marette, A. Triggering Akkermansia with dietary polyphenols: A new weapon to combat the metabolic syndrome? Gut Microbes 2016, 7, 146-153. [CrossRef]

20. Jang, S.; Sun, J.; Chen, P.; Lakshman, S.; Molokin, A.; Harnly, J.M.; Vinyard, B.T.; Urban J.F., Jr.; Davis, C.D.; Solano-Aguilar, G. Flavanol-Enriched Cocoa Powder Alters the Intestinal Microbiota, Tissue and Fluid Metabolite Profiles, and Intestinal Gene Expression in Pigs. J. Nutr. 2016, 146, 673-680. [CrossRef]

21. Magistrelli, D.; Zanchi, R.; Malagutti, L.; Galassi, G.; Canzi, E.; Rosi, F. Effects of Cocoa Husk Feeding on the Composition of Swine Intestinal Microbiota. J. Agric. Food Chem. 2016, 64, 2046-2052. [CrossRef]

22. Anhê, F.F.; Varin, T.V.; Le Barz, M.; Desjardins, Y.; Levy, E.; Roy, D.; Marette, A. Gut Microbiota Dysbiosis in Obesity-Linked Metabolic Diseases and Prebiotic Potential of Polyphenol-Rich Extracts. Curr. Obes. Rep. 2015, 4, 389-400. [CrossRef]

23. Walker, J.J.; Eckardt, P.; Aleman, J.O.; da Rosa, J.C.; Liang, Y.; Iizumi, T.; Etheve, S.; Blaser, M.J.; L Breslow, J.; Holt, P.R. The effects of trans-resveratrol on insulin resistance, inflammation, and microbiota in men with the metabolic syndrome: A pilot randomized, placebo-controlled clinical trial. J. Clin. Transl. Res. 2019, 4, 122-135.

24. Halmos, E.E.; Christophersen, C.C.; Bird, A.A.; Shepherd, S.S.; Gibson, P.P.; Muir, J.J. Diets that differ in their FODMAP content alter the colonic luminal microEnvironironment. Gut 2015, 64, 93-100. [CrossRef]

25. Halmos, E.E.; Christophersen, C.C.; Bird, A.A.; Shepherd, S.S.; Muir, J.J.; Gibson, P.P. Consistent Prebiotic Effect on Gut Microbiota With Altered FODMAP Intake in Patients with Crohn's Disease: A Randomised, Controlled Cross-Over Trial of Well-Defined Diets. Clin. Transl. Gastroenterol. 2016, 7, e164. [CrossRef]

26. Ramirez-Farias, C.; Slezak, K.; Fuller, Z.; Duncan, A.; Holtrop, G.; Louis, P. Effect of inulin on the human gut microbiota: Stimulation of Bifidobacterium adolescentis and Faecalibacterium prausnitzii. Br. J. Nutr. 2009, 101, 541-550. [CrossRef]

27. Ramnani, P.; Gaudier, E.; Bingham, M.; van Bruggen, P.; Tuohy, K.K.; Gibson, G.G. Prebiotic effect of fruit and vegetable shots containing Jerusalem artichoke inulin: A human intervention study. Br. J. Nutr. 2010, 104, 233-240. [CrossRef] 
28. Roshanravan, N.; Mahdavi, R.; Alizadeh, E.; Ghavami, A.; Rahbar Saadat, Y.; Mesri Alamdari, N.; Alipour, S.; Dastouri, M.R.; Ostadrahimi, A. The effects of sodium butyrate and inulin supplementation on angiotensin signaling pathway via promotion of Akkermansia muciniphila abundance in type 2 diabetes; A randomized, double-blind, placebo-controlled trial. J. Cardiovasc. Thorac. Res. 2017, 9, 183-190. [CrossRef]

29. Moher, D.; Liberati, A.; Tetzlaff, J.; Altman, D.D.; Group, P. Preferred reporting items for systematic reviews and meta-analyses: The PRISMA statement. PLoS Med. 2009, 6, e1000097. [CrossRef]

30. Stroup, D.F.; Berlin, J.J.; Morton, S.C.; Olkin, I.; Williamson, G.D.; Rennie, D.; Moher, D.; Becker, B.J.; Sipe, T.A.; Thacker, S.B. Meta-analysis of observational studies in epidemiology: A proposal for reporting. Meta-analysis Of Observational Studies in Epidemiology (MOOSE) group. JAMA 2008, 283, 2008-2012. [CrossRef]

31. Higgins, J.P.; Altman, D.G.; Gøtzsche, P.C.; Jüni, P.; Moher, D.; Oxman, A.D.; Savovic, J.; Schulz, K.F.; Weeks, L.; Sterne, J.A.; et al. The Cochrane Collaboration's tool for assessing risk of bias in randomised trials. BMJ 2011, 343, d5928. [CrossRef]

32. Benjamin, J.L.; Hedin, C.R.; Koutsoumpas, A.; Ng, S.C.; McCarthy, N.E.; Hart, A.L.; Kamm, M.A.; Sanderson, J.D.; Knight, S.C.; Forbes, A.; et al. Randomised, double-blind, placebo-controlled trial of fructo-oligosaccharides in active Crohn's disease. Gut 2011, 60, 923-929. [CrossRef]

33. Blatchford, P.; Stoklosinski, H.; Eady, S.; Wallace, A.; Butts, C.; Gearry, R.; Gibson, G.; Ansell, J. Consumption of kiwifruit capsules increases Faecalibacterium prausnitzii abundance in functionally constipated individuals: A randomised controlled human trial. J. Nutr. Sci. 2017, 6, e52. [CrossRef]

34. Clavel, T.; Fallani, M.; Lepage, P.; Levenez, F.; Mathey, J.; Rochet, V.; Sérézat, M.; Sutren, M.; Henderson, G.; Bennetau-Pelissero, C.; et al. Isoflavones and functional foods alter the dominant intestinal microbiota in postmenopausal women. J. Nutr. 2005, 135, 2786-2792. [CrossRef]

35. Dao, M.M.; Everard, A.; Aron-Wisnewsky, J.; Sokolovska, N.; Prifti, E.; Verger, E.O.; Kayser, B.D.; Levenez, F.; Chilloux, J.; Hoyles, L.; et al. Akkermansia muciniphila and improved metabolic health during a dietary intervention in obesity: Relationship with gut microbiome richness and ecology. Gut 2016, 65, 426-436. [CrossRef]

36. Dewulf, E.M.; Cani, P.D.; Claus, S.P.; Fuentes, S.; Puylaert, P.G.; Neyrinck, A.M.; Bindels, L.B.; de Vos, W.M.; Gibson, G.R.; Thissen, J.P.; et al. Insight into the prebiotic concept: Lessons from an exploratory, double blind intervention study with inulin-type fructans in obese women. Gut 2013, 62, 1112-1121. [CrossRef]

37. Fava, F.; Gitau, R.; Griffin, B.B.; Gibson, G.G.; Tuohy, K.K.; Lovegrove, J.J. The type and quantity of dietary fat and carbohydrate alter faecal microbiome and short-chain fatty acid excretion in a metabolic syndrome 'at-risk' population. Int. J. Obes. (2005) 2013, 37, 216-223. [CrossRef]

38. Fernando, W.W.; Hill, J.J.; Zello, G.G.; Tyler, R.R.; Dahl, W.W.; Van Kessel, A.A. Diets supplemented with chickpea or its main oligosaccharide component raffinose modify faecal microbial composition in healthy adults. Benef. Microbes 2010, 1, 197-207. [CrossRef]

39. Guadamuro, L.; Delgado, S.; Redruello, B.; Flórez, A.B.; Suárez, A.; Martínez-Camblor, P.; Mayo, B. Equol status and changes in fecal microbiota in menopausal women receiving long-term treatment for menopause symptoms with a soy-isoflavone concentrate. Front. Microbiol. 2015, 6, 777. [CrossRef]

40. Hooda, S.; Boler, B.M.; Serao, M.C.; Brulc, J.M.; Staeger, M.A.; Boileau, T.W.; Dowd, S.E.; Fahey, G.C., Jr.; Swanson, K.S. 454 pyrosequencing reveals a shift in fecal microbiota of healthy adult men consuming polydextrose or soluble corn fiber. J. Nutr. 2012, 142, 1259-1265. [CrossRef]

41. Hustoft, T.N.; Hausken, T.; Ystad, S.O.; Valeur, J.; Brokstad, K.; Hatlebakk, J.G.; Lied, G.A. Effects of varying dietary content of fermentable short-chain carbohydrates on symptoms, fecal microEnvironironment, and cytokine profiles in patients with irritable bowel syndrome. Neurogastroenterol. Motil. Off. J. Eur. Gastrointest. Motil. Soc. 2017, 29, e12969. [CrossRef]

42. Lee, T.; Clavel, T.; Smirnov, K.; Schmidt, A.; Lagkouvardos, I.; Walker, A.; Lucio, M.; Michalke, B.; Schmitt-Kopplin, P.; Fedorak, R.; et al. Oral versus intravenous iron replacement therapy distinctly alters the gut microbiota and metabolome in patients with IBD. Gut 2017, 66, 863-871. [CrossRef]

43. Li, Z.; Henning, S.M.; Lee, R.P.; Lu, Q.Y.; Summanen, P.H.; Thames, G.; Corbett, K.; Downes, J.; Tseng, C.H.; Finegold, S.M.; et al. Pomegranate extract induces ellagitannin metabolite formation and changes stool microbiota in healthy volunteers. Food Funct. 2015, 6, 2487-2495. [CrossRef]

44. Majid, H.H.; Cole, J.; Emery, P.P.; Whelan, K. Additional oligofructose/inulin does not increase faecal bifidobacteria in critically ill patients receiving enteral nutrition: A randomised controlled trial. Clin. Nutr. (Edinb. Scotl.) 2014, 33, 966-972. [CrossRef] 
45. Medina-Vera, I.; Sanchez-Tapia, M.; Noriega-Lopez, L.; Granados-Portillo, O.; Guevara-Cruz, M.; Flores-López, A.; Avila-Nava, A.; Fernández, M.L.; Tovar, A.R.; Torres, N. A dietary intervention with functional foods reduces metabolic endotoxaemia and attenuates biochemical abnormalities by modifying faecal microbiota in people with type 2 diabetes. Diabetes Metab. 2019, 45, 122-131. [CrossRef]

46. Moreno-Indias, I.; Sanchez-Alcoholado, L.; Perez-Martinez, P.; Andrés-Lacueva, C.; Cardona, F.; Tinahones, F.; Queipo-Ortuño, M.I. Red wine polyphenols modulate fecal microbiota and reduce markers of the metabolic syndrome in obese patients. Food Funct. 2016, 7, 1775-1787. [CrossRef]

47. Most, J.; Penders, J.; Lucchesi, M.; Goossens, G.G.; Blaak, E.E. Gut microbiota composition in relation to the metabolic response to 12-week combined polyphenol supplementation in overweight men and women. Eur. J. Clin. Nutr. 2017, 71, 1040-1045. [CrossRef]

48. Pinheiro, I.; Robinson, L.; Verhelst, A.; Marzorati, M.; Winkens, B.; den Abbeele, P.V.; Possemiers, S. A yeast fermentate improves gastrointestinal discomfort and constipation by modulation of the gut microbiome: Results from a randomized double-blind placebo-controlled pilot trial. BMC Complement. Altern. Med. 2017, 17, 441. [CrossRef]

49. Tagliabue, A.; Ferraris, C.; Uggeri, F.; Trentani, C.; Bertoli, S.; de Giorgis, V.; Veggiotti, P.; Elli, M. Short-term impact of a classical ketogenic diet on gut microbiota in GLUT1 Deficiency Syndrome: A 3-month prospective observational study. Clin. Nutr. ESPEN 2017, 17, 33-37. [CrossRef]

50. Wijayabahu, A.A.; Waugh, S.S.; Ukhanova, M.; Mai, V. Dietary raisin intake has limited effect on gut microbiota composition in adult volunteers. Nutr. J. 2019, 18, 14. [CrossRef]

51. Xu, J.; Lian, F.; Zhao, L.; Zhao, Y.; Chen, X.; Zhang, X.; Guo, Y.; Zhang, C.; Zhou, Q.; Xue, Z.; et al. Structural modulation of gut microbiota during alleviation of type 2 diabetes with a Chinese herbal formula. ISME J. 2015, 9, 552-562. [CrossRef]

52. Benus, R.F.; van der Werf, T.S.; Welling, G.W.; Judd, P.A.; Taylor, M.A.; Harmsen, H.J.; Whelan, K. Association between Faecalibacterium prausnitzii and dietary fibre in colonic fermentation in healthy human subjects. Br. J. Nutr. 2010, 104, 693-700. [CrossRef]

53. James, S.L.; Christophersen, C.T.; Bird, A.R.; Conlon, M.A.; Rosella, O.; Gibson, P.R.; Muir, J.G. Abnormal fibre usage in UC in remission. Gut 2015, 64, 562-570. [CrossRef]

54. Vulevic, J.; Juric, A.; Tzortzis, G.; Gibson, G.G. A mixture of trans-galactooligosaccharides reduces markers of metabolic syndrome and modulates the fecal microbiota and immune function of overweight adults1-3. J. Nutr. 2013, 143, 324-331. [CrossRef]

55. West, N.P.; Christophersen, C.T.; Pyne, D.B.; Cripps, A.W.; Conlon, M.A.; Topping, D.L.; Kang, S.; McSweeney, C.S.; Fricker, P.A.; Aguirre, D.; et al. Butyrylated starch increases colonic butyrate concentration but has limited effects on immunity in healthy physically active individuals. Exerc. Immunol. Rev. 2013, 19, 102-119.

56. Bull, M.M.; Plummer, N.N. Part 1: The Human Gut Microbiome in Health and Disease. Integr. Med. 2014, 13, 17-22.

57. Vyas, U.; Ranganathan, N. Probiotics, prebiotics, and synbiotics: Gut and beyond. Gastroenterol. Res. Pract. 2012, 2012, 872716. [CrossRef]

58. Karlsson, F.H.; Tremaroli, V.; Nookaew, I.; Bergström, G.; Behre, C.J.; Fagerberg, B.; Nielsen, J.; Bäckhed, F. Gut metagenome in European women with normal, impaired and diabetic glucose control. Nature 2013, 498, 99-103. [CrossRef]

59. Vrieze, A.; Van Nood, E.; Holleman, F.; Salojärvi, J.; Kootte, R.S.; Bartelsman, J.F.; Dallinga-Thie, G.M.; Ackermans, M.T.; Serlie, M.J.; Oozeer, R.; et al. Transfer of intestinal microbiota from lean donors increases insulin sensitivity in individuals with metabolic syndrome. Gastroenterology 2012, 143, 913-916.e7. [CrossRef]

60. Belzer, C.; Chia, L.W.; Aalvink, S.; Chamlagain, B.; Piironen, V.; Knol, J.; de Vos, W.M. Microbial Metabolic Networks at the Mucus Layer Lead to Diet-Independent Butyrate and Vitamin B12 Production by Intestinal Symbionts. MBio 2017, 8. [CrossRef]

61. Chang, P.P.; Hao, L.; Offermanns, S.; Medzhitov, R. The microbial metabolite butyrate regulates intestinal macrophage function via histone deacetylase inhibition. Proc. Natl. Acad. Sci. USA 2014, 111, 2247-2252. [CrossRef]

62. Furusawa, Y.; Obata, Y.; Fukuda, S.; Endo, T.A.; Nakato, G.; Takahashi, D.; Nakanishi, Y.; Uetake, C.; Kato, K.; Kato, T.; et al. Commensal microbe-derived butyrate induces the differentiation of colonic regulatory $\mathrm{T}$ cells. Nature 2013, 504, 446-450. [CrossRef] 
63. Kelly, C.J.; Zheng, L.; Campbell, E.L.; Saeedi, B.; Scholz, C.C.; Bayless, A.J.; Wilson, K.E.; Glover, L.E.; Kominsky, D.J.; Magnuson, A.; et al. Crosstalk between Microbiota-Derived Short-Chain Fatty Acids and Intestinal Epithelial HIF Augments Tissue Barrier Function. Cell Host Microbe 2015, 17, 662-671. [CrossRef]

64. Wang, H.; Hou, L.; Kwak, D.; Fassett, J.; Xu, X.; Chen, A.; Chen, W.; Blazar, B.R.; Xu, Y.; Hall, J.L.; et al. Increasing Regulatory T Cells With Interleukin-2 and Interleukin-2 Antibody Complexes Attenuates Lung Inflammation and Heart Failure Progression. Hypertension 2016, 68, 114-122. [CrossRef]

65. De Vadder, F.; Kovatcheva-Datchary, P.; Goncalves, D.; Vinera, J.; Zitoun, C.; Duchampt, A.; Bäckhed, F.; Mithieux, G. Microbiota-generated metabolites promote metabolic benefits via gut-brain neural circuits. Cell 2014, 156, 84-96. [CrossRef]

66. Lukovac, S.; Belzer, C.; Pellis, L.; Keijser, B.J.; de Vos, W.M.; Montijn, R.C.; Roeselers, G. Differential modulation by Akkermansia muciniphila and Faecalibacterium prausnitzii of host peripheral lipid metabolism and histone acetylation in mouse gut organoids. MBio 2014, 5. [CrossRef]

67. Lee, H.; Ko, G. Effect of metformin on metabolic improvement and gut microbiota. Appl. Environ. Microbiol. 2014, 80, 5935-5943. [CrossRef]

68. Shin, N.R.; Lee, J.C.; Lee, H.Y.; Kim, M.S.; Whon, T.W.; Lee, M.S.; Bae, J.W. An increase in the Akkermansia spp. population induced by metformin treatment improves glucose homeostasis in diet-induced obese mice. Gut 2014, 63, 727-735. [CrossRef]

69. Everard, A.; Belzer, C.; Geurts, L.; Ouwerkerk, J.P.; Druart, C.; Bindels, L.B.; Guiot, Y.; Derrien, M.; Muccioli, G.G.; Delzenne, N.M.; et al. Cross-talk between Akkermansia muciniphila and intestinal epithelium controls diet-induced obesity. Proc. Natl. Acad. Sci. USA 2013, 110, 9066-9071. [CrossRef]

70. Singh, R.K.; Chang, H.W.; Yan, D.; Lee, K.M.; Ucmak, D.; Wong, K.; Abrouk, M.; Farahnik, B.; Nakamura, M.; Zhu, T.H.; et al. Influence of diet on the gut microbiome and implications for human health. J. Transl. Med. 2017, 15. [CrossRef]

71. Morrison, D.D.; Preston, T. Formation of short chain fatty acids by the gut microbiota and their impact on human metabolism. Gut Microbes 2016, 7, 189-200. [CrossRef]

72. Jardine, M. Nutrition Considerations for Microbiota Health in Diabetes. Diabetes Spectr. A Publ. Am. Diabetes Assoc. 2016, 29, 238-244. [CrossRef]

73. Liu, X.; Wu, Y.; Li, F.; Zhang, D. Dietary fiber intake reduces risk of inflammatory bowel disease: Result from a meta-analysis. Nutr. Res. 2015, 35, 753-758. [CrossRef]

74. McRae, M.M. Dietary Fiber Intake and Type 2 Diabetes Mellitus: An Umbrella Review of Meta-analyses. J. Chiropr. Med. 2018, 17, 44-53. [CrossRef]

75. Ismail, T.; Sestili, P.; Akhtar, S. Pomegranate peel and fruit extracts: A review of potential anti-inflammatory and anti-infective effects. J. Ethnopharmacol. 2012, 143, 397-405. [CrossRef]

76. Medjakovic, S.; Jungbauer, A. Pomegranate: A fruit that ameliorates metabolic syndrome. Food Funct. 2013, 4, 19-39. [CrossRef]

77. Franco, O.H.; Chowdhury, R.; Troup, J.; Voortman, T.; Kunutsor, S.; Kavousi, M.; Oliver-Williams, C.; Muka, T. Use of Plant-Based Therapies and Menopausal Symptoms: A Systematic Review and Meta-analysis. JAMA 2016, 315, 2554-2563. [CrossRef]

78. Glisic, M.; Kastrati, N.; Gonzalez-Jaramillo, V.; Bramer, W.M.; Ahmadizar, F.; Chowdhury, R.; Danser, A.J.; Roks, A.J.; Voortman, T.; Franco, O.H.; et al. Associations between Phytoestrogens, Glucose Homeostasis, and Risk of Diabetes in Women: A Systematic Review and Meta-Analysis. Adv. Nutr. 2018, 9, 726-740. [CrossRef]

79. Ozdal, T.; Sela, D.D.; Xiao, J.; Boyacioglu, D.; Chen, F.; Capanoglu, E. The Reciprocal Interactions between Polyphenols and Gut Microbiota and Effects on Bioaccessibility. Nutrients 2016, 8, 78. [CrossRef]

80. David, L.A.; Maurice, C.F.; Carmody, R.N.; Gootenberg, D.B.; Button, J.E.; Wolfe, B.E.; Ling, A.V.; Devlin, A.S.; Varma, Y.; Fischbach, M.A.; et al. Diet rapidly and reproducibly alters the human gut microbiome. Nature 2014, 505, 559-563. [CrossRef]

81. Gomez, A.; Luckey, D.; Taneja, V. The gut microbiome in autoimmunity: Sex matters. Clin. Immunol. 2015, 159, 154-162. [CrossRef]

82. Kaplan, H.; Hill, K.; Lancaster, J.; Hurtado, A.A. A theory of human life history evolution: Diet, intelligence, and longevity. Evol. Anthropol. Issues News Rev. 2000, 9, 156-185. [CrossRef] 
83. Zeevi, D.; Korem, T.; Zmora, N.; Israeli, D.; Rothschild, D.; Weinberger, A.; Ben-Yacov, O.; Lador, D.; Avnit-Sagi, T.; Lotan-Pompan, M.; et al. Personalized Nutrition by Prediction of Glycemic Responses. Cell 2015, 163, 1079-1094. [CrossRef]

84. Moen, B.; Berget, I.; Rud, I.; Hole, A.A.; Kjos, N.N.; Sahlstrom, S. Extrusion of barley and oat influence the fecal microbiota and SCFA profile of growing pigs. Food Funct. 2016, 7, 1024-1032. [CrossRef]

85. Grundy, M.M.-L.; Lapsley, K.; Ellis, P.P. A review of the impact of processing on nutrient bioaccessibility and digestion of almonds. Int. J. Food Sci. Technol. 2016, 51, 1937-1946. [CrossRef]

86. Zinöcker, M.M.; Lindseth, I.I. The Western Diet-Microbiome-Host Interaction and Its Role in Metabolic Disease. Nutrients 2018, 10, 365. [CrossRef]

(C) 2019 by the authors. Licensee MDPI, Basel, Switzerland. This article is an open access article distributed under the terms and conditions of the Creative Commons Attribution (CC BY) license (http://creativecommons.org/licenses/by/4.0/). 